\title{
On Local Antimagic Chromatic Number of Spider Graphs
}

\author{
Gee-Choon Lau ${ }^{a}$, Wai-Chee Shiu ${ }^{b, c}$, Chee-Xian Soo ${ }^{d}$ \\ ${ }^{a}$ Faculty of Computer $\&$ Mathematical Sciences, \\ Universiti Teknologi MARA (Segamat Campus), \\ 85000, Johor, Malaysia. \\ geeclau@yahoo.com \\ ${ }^{b}$ Department of Mathematics, The Chinese University of Hong Kong, \\ Shatin, Hong Kong. \\ ${ }^{c}$ College of Global Talents, Beijing Institute of Technology, \\ Zhuhai, China. \\ wcshiu@associate.hkbu.edu.hk \\ ${ }^{d}$ School of Physical and Mathematical Sciences, \\ Nanyang Technological University, 21 Nanyang Link, \\ 637731, Singapore. \\ csoo002@e.ntu.edu.sg
}

\begin{abstract}
An edge labeling of a connected graph $G=(V, E)$ is said to be local antimagic if it is a bijection $f: E \rightarrow\{1, \ldots,|E|\}$ such that for any pair of adjacent vertices $x$ and $y, f^{+}(x) \neq f^{+}(y)$, where the induced vertex label $f^{+}(x)=\sum f(e)$, with $e$ ranging over all the edges incident to $x$. The local antimagic chromatic number of $G$, denoted by $\chi_{l a}(G)$, is the minimum number of distinct induced vertex labels over all local antimagic labelings of $G$. In this paper, we first show that a $d$-leg spider graph has $d+1 \leq \chi_{l a} \leq d+2$. We then obtain many sufficient conditions such that both the values are attainable. Finally, we show that each 3-leg spider has $\chi_{l a}=4$ if not all legs are of odd length. We conjecture that almost all $d$-leg spiders of size $q$ that satisfies $d(d+1) \leq 2(2 q-1)$ with each leg length at least 2 has $\chi_{l a}=d+1$.
\end{abstract}

Keywords: Local antimagic labeling, Local antimagic chromatic number, Spiders 2010 AMS Subject Classifications: 05C78; 05C69.

\section{Introduction}

A connected graph $G=(V, E)$ is said to be local antimagic if it admits a local antimagic (edge) labeling, i.e., a bijection $f: E \rightarrow\{1, \ldots,|E|\}$ such that the induced vertex labeling $f^{+}: V \rightarrow \mathbb{Z}$ given by $f^{+}(x)=\sum f(e)$ (with $e$ ranging over all the edges incident to $x$ ) has the property that any two adjacent vertices have distinct induced vertex labels. The number of distinct induced vertex labels under $f$ is denoted by $c(f)$, and is called the color number of $f$. Also, $f$ is call a local antimagic $c(f)$-labeling of $G$. The local antimagic chromatic number of $G$, denoted by $\chi_{l a}(G)$, is $\min \{c(f): f$ is a local antimagic labeling of $G\}$ (see [1 $[$ ] $]$ ). For integers $a<b$, we let $[a, b]=\{a, a+1, a+2, \ldots, b\}$.

The following two results in [5] are needed.

Lemma 1.1. Let $G$ be a graph of size $q$ containing d pendants. Let $f$ be a local antimagic labeling of $G$ such that $f(e)=q$. If $e$ is not a pendant edge, then $c(f) \geq d+2$.

\footnotetext{
${ }^{1}$ Corresponding author.
} 
Theorem 1.2. Let $G$ be a graph having $d$ pendants. If $G$ is not $K_{2}$, then $\chi_{l a}(G) \geq d+1$ and the bound is sharp.

For $1 \leq i \leq t, a_{i}, n_{i} \geq 1$ and $d=\sum_{i=1}^{t} n_{i} \geq 3$, a spider of $d$ legs, denoted $S p\left(a_{1}^{\left[n_{1}\right]}, a_{2}^{\left[n_{2}\right]}, \ldots, a_{t}^{\left[n_{t}\right]}\right.$ ) (or $S p\left(y_{1}, y_{2}, \ldots, y_{d}\right)$ for convenience), is a tree formed by identifying an end-vertex of $n_{i}$ path(s) of length $a_{i}$. The vertex $u$ of degree $d$ is the core of the spider. Note that, $S p\left(1^{[d]}\right)$ is the star graph of $d$ pendant vertices with $\chi_{l a}\left(S p\left(1^{[d]}\right)\right)=d+1$. In this paper, we first show that $d+1 \leq \chi_{l a}\left(S p\left(y_{1}, y_{2}, \ldots, y_{d}\right)\right) \leq d+2$. We then obtain many sufficient conditions such that both the values are attainable. Finally, we show that $\chi_{l a}\left(S p\left(y_{1}, y_{2}, y_{3}\right)\right)=4$ if not all $y_{1}, y_{2}, y_{3}$ are odd. We conjecture that each $d$-leg spider of size $q$ that satisfies $d(d+1) \leq 2(2 q-1)$ with each leg length at least 2 has $\chi_{l a}=d+1$ except $S p\left(2^{[n]}, 3^{[m]}\right)$ for $(n, m) \in\{(4,0),(5,0),(6,0),(0,10),(1,8),(1,9),(2,7),(2,8),(3,5),(3,6),(4,4),(4,5),(5,3)\}$.

\section{Spider Graphs}

In [6], the authors gave a family of $d$-leg spiders to have $\chi_{l a}>d+1$.

Theorem 2.1. For $d \geq 3$,

$$
\chi_{l a}\left(S p\left(2^{[d]}\right)\right)= \begin{cases}d+2 & \text { if } d \geq 4 \\ d+1 & \text { if } d=3 .\end{cases}
$$

Theorem 2.2. Let $G$ be a graph of size $q$ with $k \geq 1$ pendant vertices. Suppose $G$ has only one vertex of maximum degree $\Delta$ which is not adjacent to any pendant vertex and all other vertices of $G$ has degree at most $m<\Delta$. If $\Delta(\Delta+1)>m(2 q-m+1)$, then $\chi_{l a}(G) \geq k+2$.

Proof. Let $f$ be a local antimagic labeling of $G$. If $q$ is assigned to a non-pendant edge, by Lemma 1.1, $c(f) \geq m+2$. Assume $q$ is assigned to a pendant edge that has a non-pendant end-vertex $x$. Now, all the $k$ pendant vertex labels of $G$ are distinct and at most $q$. Suppose $u$ is a vertex of degree $\Delta$, then $f^{+}(u) \geq$ $\Delta(\Delta+1) / 2$ and that $q+1 \leq f^{+}(x) \leq m(2 q-m+1) / 2$. By the given hypothesis, $f^{+}(u)>f^{+}(x)>f^{+}(y)$ for every pendant vertex $y$. Thus, $c(f) \geq k+2$. The theorem holds.

Note that if $G$ has at least two vertices of maximum degree $\Delta$, the condition $\Delta(\Delta+1)>\Delta(2 q-\Delta+1)$ implies that $\Delta>q$, which is impossible. Also note that the conditions of Theorem 2.2 is not necessary to have $\chi_{l a}(G) \geq k+2$. A counterexample of non-tree graph is $\chi_{l a}\left(K_{3} \odot O_{2}\right)=9$, and counterexamples of trees are $S p\left(2^{[n]}\right), n=4,5,6$.

Theorem 2.3. A spider of $d \geq 3$ legs that has at least a leg of length 1 has $\chi_{l a}=d+1$.

Proof. Let $G=S p\left(a_{1}, a_{2}, \ldots, a_{r}, 1^{[t]}\right)$ such that $r \geq 1, t \geq 1, a_{i} \geq 2$, and $d=r+t \geq 3$. Thus, $G$ is of size $q=\sum_{i=1}^{r} a_{i}+t$. Arrange all the paths of lengths $a_{1}, a_{2}, \ldots, a_{r}$ horizontally from left to right, and name the edges from left to right as $e_{1}, e_{2}, \ldots, e_{q-t}$. Let $E=\left\{e_{j} \mid 1 \leq j \leq q-t\right\}$. Define $f: E \rightarrow[1, q-t]$ such that $f\left(e_{j}\right)=j / 2$ for even $j$ and $f\left(e_{j}\right)=(q-t)-(j-1) / 2$ for odd $j$. Finally, label the edges of the remaining $t$ path(s) of length 1 by $q-t+1$ to $q$ bijectively.

Identify all the $r+t$ right end-vertices of the paths as the core. We now have the graph $G$ with an induced edge labeling given by $f: E(G) \rightarrow[1, q]$. Observe that

(i) each degree 2 vertex has induced label $j / 2+(q-t)-j / 2=q-t$ or $(q-t)-(j-1) / 2+(j+1) / 2=q-t+1$. 
(ii) each of the $t$ pendant vertices of paths length 1 has induced label $q-t+1, \ldots, q$ respectively.

(iii) the pendant vertex of path length $a_{1}$ has induced label $q-t$ while all remaining $r-1$ pendant vertices have mutually distinct induced label at most $q-t-1$.

(iv) the core has induced label larger than $q$.

Thus, $f$ is a local antimagic labeling with $c(f)=r+t+1=d+1$ and $\chi_{l a}(G) \leq d+1$. By Theorem 1.2, $\chi_{l a}(G) \geq d+1$. The theorem holds.

Example 2.1: In the following figure, we give the above defined labeling for $S p(4,2,3,5,1)$.

\begin{tabular}{|c|c|c|c|c|}
\hline (14) 14 (15) 1 (14) 13 (15) 2 (37) & 14 & 1 & 13 & 2 \\
\hline (12) 12 (15) $3-(37)$ & 12 & 3 & & \\
\hline (11) 11 (15) 4 (14) $\frac{10}{(37)}$ & 11 & 4 & 10 & \\
\hline (5) 5 (14) 9 (15) 6 (14) 8 (15) 7 (37) & $\begin{array}{c}5 \\
15 \\
(15,14,12,11,5)\end{array}$ & 9 & 6 & 8 \\
\hline
\end{tabular}

The right vertex of each path are identified as the core $u$. We use the right table to denote this labeling together with the induced vertex labels. We shall also use such kind of table to represent a labeling for a spider in the rest of the paper.

Call the labeling of the paths of length $a_{i} \geq 2,1 \leq i \leq r$ in Theorem 2.3 the fundamental labeling of spider. By using this labeling, it is easy to get the following result:

Corollary 2.4. Suppose $G$ is a spider with $d \geq 3$ legs, then $d+1 \leq \chi_{l a}(G) \leq d+2$.

In what follows, we assume every leg of spider is of length at least 2.

Theorem 2.5. Suppose $y_{1}, y_{2}, \ldots, y_{d} \geq 2$ and $d \geq 3$. If $d(d+1)>2(2 q-1)$, then $\chi_{l a}\left(S p\left(y_{1}, y_{2}, \ldots, y_{d}\right)\right)=$ $d+2$.

Proof. Let $g$ be a local antimagic labeling of $G=S p\left(y_{1}, y_{2}, \ldots, y_{d}\right)$. Under the hypothesis, by Theorem 2.2, we know $c(g) \geq d+2$. By Corollary 2.4, the theorem holds.

Corollary 2.6. There are infinitely many spiders of $d \geq 3$ legs with $\chi_{l a}=d+2$.

Theorem 2.7. For $n \geq 0, m \geq 1$ and $n+m \geq 3, \chi_{l a}\left(S p\left(2^{[n]}, 3^{[m]}\right)\right)=n+m+2$ if $(n, m) \notin A$, where

$$
\begin{aligned}
A= & \left\{\left(0, i_{0}\right) \mid i_{0} \in[3,10]\right\} \cup\left\{\left(1, i_{1}\right) \mid i_{1} \in[2,9]\right\} \cup\left\{\left(2, i_{2}\right) \mid i_{2} \in[1,8]\right\} \cup\left\{\left(3, i_{3}\right) \mid i_{3} \in[1,6]\right\} \\
& \cup\left\{\left(4, i_{4}\right) \mid i_{4} \in[1,5]\right\} \cup\{(5,1),(5,2),(5,3),(6,1)\} .
\end{aligned}
$$

Proof. Let $G=S p\left(2^{[n]}, 3^{[m]}\right)$. Now, $q=2 n+3 m$ and $d=n+m$. The inequality $(n+m)(n+m+1) \leq$ $2(4 n+6 m-1)$ is equivalent to $\left(n+m-\frac{7}{2}\right)^{2} \leq 4 m+\frac{41}{4}$.

If $m \geq 11$, then $\left(n+m-\frac{7}{2}\right)^{2} \geq\left(m-\frac{7}{2}\right)^{2}=m^{2}-7 m+\frac{49}{4}>4 m+\frac{41}{4}$.

If $n \geq 7$, then $\left(n+m-\frac{7}{2}\right)^{2} \geq m^{2}+7 m+\frac{49}{4}>4 m+\frac{41}{4}$.

So we have to deal with $1 \leq m \leq 10$ and $0 \leq n \leq 6$. It is routine to check that $(n+m)(n+m+1) \leq$ $2(4 n+6 m-1)$ if and only if $(n, m) \in A$. In other word, $(n, m) \notin A$ if and only if $d(d+1)>2(2 q-1)$. By Theorem 2.5, we know that $\chi_{l a}(G)=n+m+2$ if $(n, m) \notin A$. Thus, the theorem holds. 
Let us analyze the graph $G=S p\left(2^{[n]}, 3^{[m]}\right)$, where $(n, m) \in A$ and $A$ is defined in Theorem 2.7. By Appendix, it suffices to consider $(n, m) \in\{(0,10),(1,8),(1,9),(2,7),(2,8),(3,5),(3,6),(4,4),(4,5),(5,3)\}$.

Denote the legs of length 2 as $P_{3}^{(i)}=x_{i 1} x_{i 2} x_{i 3}, 1 \leq i \leq n$ and those of length 3 as $P_{4}^{(j)}=y_{j 1} y_{j 2} y_{j 3} y_{j 4}$, $1 \leq j \leq m$. Also the core $u=x_{i 3}=y_{j 4}$ for all $i, j$. Let $f$ be a local antimagic $(m+n+1)$-labeling. Note that, $f^{+}(u) \geq \frac{1}{2}(n+m)(n+m+1) \geq 4 n+4 m+4>2 n+3 m=q$.

By Lemma 1.1, $q$ is labeled at a pendant edge. Suppose $q=2 n+3 m$ is labeled at a pendant edge of a leg of length $2(n \geq 1)$. Without loss of generality, we may assume $f\left(x_{11} x_{12}\right)=q$. Since $f^{+}\left(x_{12}\right) \geq q+1$ and $f^{+}\left(x_{i 1}\right)$ and $f^{+}\left(y_{j 1}\right)$ are at most $q$ for $1 \leq i \leq n$ and $1 \leq j \leq m, f^{+}\left(x_{13}\right)=f^{+}(u) \leq q$ which is a contradiction. So $q$ must be labeled at a pendant edge of a leg of length 3. By symmetric we may assume that $f\left(y_{11} y_{12}\right)=q$. Then, $f^{+}\left(y_{11}\right)=q, f^{+}\left(y_{12}\right) \geq q+1$ and the other induced vertex colors are less than $q$. Thus $f^{+}(u)=f^{+}\left(y_{12}\right)$. Let $f\left(y_{12} y_{13}\right)=x$. Then $f^{+}(u)=f^{+}\left(y_{12}\right)=q+x$. Then $q+x \geq \sum_{i=1}^{m+n} i=\frac{1}{2}(m+n)(m+n+1)$. So

$$
x \in\left[\frac{1}{2}(n+m)(n+m+1)-(2 n+3 m), 2 n+3 m-1\right] .
$$

(A) Suppose $n=0$. Only the case $(n, m)=(0,10)$. From (2.1) we have $x \in[25,29]$. Following table lists all the possible labels assigned at the edges incident to the core:

\begin{tabular}{c|c|l}
$x$ & $f^{+}(u)$ & labels incident to the core \\
\hline 25 & 55 & $1,2,3,4,5,6,7,8,9,10$ \\
26 & 56 & $1,2,3,4,5,6,7,8,9,11$ \\
27 & 57 & $1,2,3,4,5,6,7,8,10,11$ \\
27 & 57 & $1,2,3,4,5,6,7,8,9,12$ \\
28 & 58 & $1,2,3,4,5,6,7,8,9,13$ \\
28 & 58 & $1,2,3,4,5,6,7,8,10,12$ \\
28 & 58 & $1,2,3,4,5,6,7,9,10,11$ \\
29 & 59 & $1,2,3,4,5,6,7,8,9,14$ \\
29 & 59 & $1,2,3,4,5,6,7,8,10,13$ \\
29 & 59 & $1,2,3,4,5,6,7,9,10,12$ \\
29 & 59 & $1,2,3,4,5,6,8,9,10,11$
\end{tabular}

Note that integers in $[1,6]$ are labeled to edges incident to the core (we shall say that the labels are incident to the core). So, the edge with label 24 must be adjacent to an edge with label $s, 7 \leq s \leq 29$. Thus the incident vertex of these two labels must have label $q+x$. This means $s=q+x-24=x+6 \geq 31$ which is impossible. Thus $\chi_{l a}\left(S p\left(3^{[10]}\right)\right)=12$.

(B) Suppose $n=1$. There are two cases $(n, m)=(1,8),(1,9)$. When $(n, m)=(1,8)$. From (2.1) we have $x \in[19,25]$. It is easy to check that labels in $[1,28-x]$ are incident to the core. Since there is only one leg of length 2 , there exists $y \in\{x-1, x-2\}$ is labeled at an edge of the leg of length 3 . Since labels in $[1,28-x]$ are incident to the core, similar to Case (A), we get that $q+x-y \geq q+1$. So, there is no local antimagic 10-labeling for $S p\left(2,3^{[8]}\right)$. Thus, $\chi_{l a}\left(S p\left(2,3^{[8]}\right)\right)=11$.

When $(n, m)=(1,9)$. From (2.1) we have $x \in[26,27]$. By a similar argument as above, we get that $\chi_{l a}\left(S p\left(2,3^{[9]}\right)\right)=12$.

(C) Suppose $n=2$. There are two cases $(n, m)=(2,7),(2,8)$.

When $(n, m)=(2,7)$. From (2.1) we have $x \in[20,24]$. It is easy to check that labels in $[1,29-x]$ are incident to the core. Since there is only two legs of length 2 , there exists $y \in\{x-1, x-2, x-3\}$ is 
labeled at an edge of the leg of length 3. Since labels in $[1,29-x]$ are incident to the core, similar to Case (A), we get that $q+x-y \geq q+1$. So, there is no local antimagic 10-labeling for $S p\left(2^{[2]}, 3^{[7]}\right)$. Thus, $\chi_{l a}\left(S p\left(2^{[2]}, 3^{[7]}\right)\right)=11$.

When $(n, m)=(2,8)$. From (2.1) we have $x=27$. By a similar argument, we have $\chi_{l a}\left(S p\left(2^{[2]}, 3^{[8]}\right)\right)=$ 12 .

(D) For the case $(n, m) \in\{(3,5),(3,6),(4,4),(4,5),(5,3)\}$. The proof is similar to the above cases. So we omit here.

Hence we have,

Theorem 2.8. For $n \geq 0, m \geq 1$ and $n+m \geq 3, \chi_{l a}\left(S p\left(2^{[n]}, 3^{[m]}\right)\right)=n+m+1$ if and only if $(n, m) \in B$, where

$$
\begin{aligned}
B= & \left\{\left(0, i_{0}\right) \mid i_{0} \in[3,9]\right\} \cup\left\{\left(1, i_{1}\right) \mid i_{1} \in[2,7]\right\} \cup\left\{\left(2, i_{2}\right) \mid i_{2} \in[1,6]\right\} \cup\left\{\left(3, i_{3}\right) \mid i_{3} \in[1,4]\right\} \\
& \cup\left\{\left(4, i_{4}\right) \mid i_{4} \in[1,3]\right\} \cup\{(5,1),(5,2),(6,1)\} .
\end{aligned}
$$

Theorem 2.9. Let $y_{1}, \ldots, y_{d} \geq 2$ be evens such that $y_{d}=y_{d-2}+2 y_{d-3}+\cdots+(d-3) y_{2}+(d-2) y_{1}$. Then $\chi_{l a}\left(S p\left(y_{1}, \ldots, y_{d}\right)\right)=d+1$, where $d \geq 2$.

Proof. Let $G=S p\left(y_{1}, y_{2}, \ldots, y_{d}\right)$ that satisfies the given conditions. Note that, the size of $G$ is $q=\sum_{i=1}^{d} y_{i}$ and the condition can be rewritten as $\sum_{i=1}^{d}(d-i-1) y_{i}=0$. Apply the fundamental labeling of spider to $G$. Identify all the right end-vertices (with label $\frac{1}{2}\left(y_{1}+\cdots+y_{i}\right)$ ) for path of length $y_{i}$. We now have the graph $G$ with an induced edge labeling $f: E(G) \rightarrow[1, q]$ such that

(i) each degree 2 vertex has induced label $i / 2+q-i / 2=q$ or $q-(i-1) / 2+(i+1) / 2=q+1$. Moreover, the induced label of the second or the last second vertex of each path is $q+1$.

(ii) the end-vertex incident to edge $e_{1}$ has induced label $q$ and the remaining $d-1$ end-vertices have mutually distinct induced labels at least $q / 2+1$ and at most $q-1$.

(iii) the core has induced label

$$
\begin{aligned}
f^{+}(u) & =y_{1} / 2+\left(y_{1}+y_{2}\right) / 2+\left(y_{1}+y_{2}+y_{3}\right) / 2+\cdots+\left(y_{1}+y_{2}+\cdots+y_{d}\right) / 2 \\
& =\frac{1}{2} \sum_{i=1}^{d}(d-i+1) y_{i}=\frac{1}{2} \sum_{i=1}^{d}(d-i-1) y_{i}+q \\
& =q .
\end{aligned}
$$

Thus, $f$ is a local antimagic labeling with $c(f)=d+1$ and $\chi_{l a}(G) \leq d+1$. Hence, the theorem holds by Theorem 1.2 . 
Example 2.2: Consider $\operatorname{Sp}(2,4,6,4,20)$ which satisfies the hypothesis of Theorem 2.9, According to the fundamental labeling we have:

36,1 ;

$35,2,34,3$;

$33,4,32,5,31,6$;

$30,7,29,8$;

$28,9,27,10,26,11,25,12,24,13,23,14,22,15,21,16,20,17,19,18$.

$(37,36,36,33,30)$

Corollary 2.10. Let $y_{1}, \ldots, y_{d} \geq 2$ be evens. If $\sum_{i=1}^{d-1}(d-i) y_{i}=\sum_{i=k+1}^{d} y_{i}$, where $0 \leq k \leq d-1$, then $\chi_{l a}\left(\operatorname{Sp}\left(y_{1}, \ldots, y_{d}\right)\right)=d+1$.

Proof. Keep the notation in the proof of Theorem [2.9. From the labeling $f$, we have known that each pendant vertex has induced label $q-\frac{1}{2} \sum_{i=1}^{k} y_{i}, 0 \leq k \leq d-1$. Note that when $k=0$ the empty summation is treated as zero. Thus, $\chi_{l a}\left(S p\left(y_{1}, \ldots, y_{d}\right)=d+1\right.$ if $f^{+}(u)=q-\frac{1}{2} \sum_{i=1}^{k} y_{i}$ for some $0 \leq k \leq d-1$. From (2.2), we have $\frac{1}{2} \sum_{i=1}^{d}(d-i-1) y_{i}+q=q-\frac{1}{2} \sum_{i=1}^{k} y_{i}$. It is equivalent to $\sum_{i=1}^{d}(d-i) y_{i}=\sum_{i=1}^{d} y_{i}-\sum_{i=1}^{k} y_{i}$ or $\sum_{i=1}^{d-1}(d-i) y_{i}=\sum_{i=k+1}^{d} y_{i}$

Corollary 2.11. Let $y_{1}, \ldots, y_{d} \geq 2$ be evens. If $\sum_{i=1}^{d-1}(d-i) y_{i}=\sum_{i=k+1}^{d} y_{i}$, where $1 \leq k \leq d-1$, then $\chi_{l a}\left(S p\left(y_{1}, \ldots, y_{d}+1\right)\right)=d+1$.

Example 2.3: $\quad$ Consider $y_{1}=4, y_{2}=2, y_{3}=4, y_{4}=2$.

1. By Theorem 2.9 or Corollary 2.10 with $k=0$, we get $y_{5}=20$ and so $\chi_{l a}(\operatorname{Sp}(4,2,4,2,20))=6$. According to the fundamental labeling we get the following labeling of each leg:

$32,1,31,2$;

30,3 ;

$29,4,28,5$;

27,6 ;

$26,7,25,8,24,9,23,10,22,11,21,12,20,13,19,14,18,15,17,16$.

$(33,32,30,29,27,26)$

2. By Corollary 2.10 with $k=1$, we get $y_{5}=24$ and so $\chi_{l a}(S p(4,2,4,2,24))=6$. According to the fundamental labeling we get the following labeling of each leg:

$36,1,35,2$, ;

34,3 ;

$33,4,32,5$;

31,6 ;

$30,7,29,8,28,9,27,10,26,11,25,12,24,13,23,14,22,15,21,16,20,17,19,18$.

$(37,36,34,33,31,30)$ 
3. By Corollary 2.11, from Case $2, \chi_{l a}(S p(4,2,4,2,25))=6$. According to the fundamental labeling we get the following labeling of each leg:

$37,1,36,2$, ;

35,3 ;

$34,4,33,5$;

32,6 ;

$31,7,30,8,29,9,28,10,27,11,26,12,25,13,24,14,23,15,22,16,21,17,20,18,19$.

$(38,37,35,34,32,31)$

By Corollary 2.11 with $k=4$, we also get $\chi_{l a}(S p(4,2,4,2,33))=6$.

Example 2.4: Let $y_{1}=4 y_{2}=6$. Using $k=1,2 y_{1}+y_{2}=14=y_{2}+y_{3}$ gives $y_{3}=8$. So, we get $S p(4,6,8)$ with the following labeling.

$18,1,17,2$;

$16,3,15,4,14,5$;

$13,6,12,7,11,8,10,9$

$(19,18,16,13)$

By Corollary 2.11, we also obtain $\chi_{l a}(S p(4,6,13))=\chi_{l a}(S p(4,6,19))=4$.

Corollary 2.12. If $a, b \geq 2$ are even, then $\left.\chi_{l a}(S p(a, b, a))=\chi_{l a}(S p(a, b, 2 a))=\chi_{l a} S p(a, b, 2 a+b)\right)=$ $\left.\chi_{l a}(S p(a, b, a+1))=\chi_{l a}(S p(a, b, 2 a+1))=\chi_{l a} S p(a, b, 2 a+b+1)\right)=4$.

\section{Spiders with 3 legs}

Before considering some labelings of spider of 3 legs, we show some useful labelings for a path first.

Lemma 3.1. Suppose $N \geq 2$ and

$$
r \in \begin{cases}\{1\}, & N=2 ; \\ \{2 j \mid 1 \leq j \leq N / 2-1\} \cup\{1, N-1\}, & \text { even } N \geq 4 \\ \{2 j-1 \mid 1 \leq j \leq(N-1) / 2\} \cup\{N-1\}, & \text { odd } N\end{cases}
$$

There is a circular permutation $\left(a_{i}\right)_{i=1}^{N}$ of $[1, N]$ such that the sum of two consecutive terms $a_{i}+a_{i+1} \in$ $\{N, N+1, N+2\}$ for $1 \leq i \leq N$ and $\left|a_{N}-a_{1}\right|=r$, where the indices of $a_{i}$ 's are taken modulo $N$.

Proof. We separate into 4 cases.

(a) $N=4 k, k \geq 1$.

\begin{tabular}{|c|c|c|c|c|c|c|c|c|}
$i$ & 1 & 2 & 3 & 4 & $\cdots$ & $2 k-2$ & $2 k-1$ & $2 k$ \\
\hline \hline$a_{i}$ & $2 k$ & $2 k+2$ & $2 k-2$ & $2 k+4$ & $\cdots$ & $4 k-2$ & 2 & $4 k$ \\
\hline$a_{2 k+i}$ & 1 & $4 k-1$ & 3 & $4 k-3$ & $\cdots$ & $2 k+3$ & $2 k-1$ & $2 k+1$ \\
\hline
\end{tabular}

The set of difference of two consecutive terms of the obtained sequence is $\left\{\left|a_{i}-a_{i+1}\right|: 1 \leq i \leq 4 k\right\}=$ $\{2 j \mid 1 \leq j \leq 2 k-1\} \cup\{1,4 k-1\}$. When we shift suitably the subscripts of $a_{i}$ we will obtain that $\left|a_{N}-a_{1}\right|=r$. For the remaining cases, we will not point out this arrangement again. 
(b) $N=4 k+1, k \geq 1$.

\begin{tabular}{|c|c|c|c|c|c|c|c|c|c|}
$i$ & 1 & 2 & 3 & 4 & $\cdots$ & $2 k-2$ & $2 k-1$ & $2 k$ & $2 k+1$ \\
\hline \hline$a_{i}$ & $2 k+2$ & $2 k-1$ & $2 k+4$ & $2 k-3$ & $\cdots$ & 3 & $4 k$ & 1 & $\varnothing$ \\
\hline$a_{2 k+i}$ & $4 k+1$ & 2 & $4 k-1$ & 4 & $\cdots$ & $2 k-2$ & $2 k+3$ & $2 k$ & $2 k+1$ \\
\hline
\end{tabular}

The set of difference of two consecutive terms of the obtained sequence is $\left\{\left|a_{i}-a_{i+1}\right|: 1 \leq i \leq\right.$ $4 k+1\}=\{2 j-1 \mid 1 \leq j \leq 2 k\} \cup\{4 k\}$.

(c) $N=4 k+2, k \geq 0$.

\begin{tabular}{|c|c|c|c|c|c|c|c|c|}
$i$ & 1 & 2 & 3 & 4 & $\cdots$ & $2 k-1$ & $2 k$ & $2 k+1$ \\
\hline \hline$a_{i}$ & $2 k+1$ & $2 k+3$ & $2 k-1$ & $2 k+5$ & $\cdots$ & 3 & $4 k+1$ & 1 \\
\hline$a_{2 k+i+1}$ & $4 k+2$ & 2 & $4 k$ & 4 & $\cdots$ & $2 k+4$ & $2 k$ & $2 k+2$ \\
\hline
\end{tabular}

The set of difference of two consecutive terms of the obtained sequence is $\left\{\left|a_{i}-a_{i+1}\right|: 1 \leq i \leq\right.$ $4 k+2\}=\{2 j \mid 1 \leq j \leq 2 k\} \cup\{1,4 k+1\}$, where $k \geq 1$. When $k=0$, this set is $\{1\}$.

(d) $N=4 k+3, k \geq 0$.

\begin{tabular}{|c|c|c|c|c|c|c|c|c|c|}
$i$ & 1 & 2 & 3 & 4 & $\cdots$ & $2 k-1$ & $2 k$ & $2 k+1$ & $2 k+2$ \\
\hline \hline$a_{i}$ & $2 k+1$ & $2 k+4$ & $2 k-1$ & $2 k+6$ & $\cdots$ & 3 & $4 k+2$ & 1 & $\varnothing$ \\
\hline$a_{2 k+i+1}$ & $4 k+3$ & 2 & $4 k+1$ & 4 & $\cdots$ & $2 k+5$ & $2 k$ & $2 k+3$ & $2 k+2$ \\
\hline
\end{tabular}

The set of difference of two consecutive terms of the obtained sequence is $\left\{\left|a_{i}-a_{i+1}\right|: 1 \leq i \leq\right.$ $4 k+3\}=\{2 j-1 \mid 1 \leq j \leq 2 k+1\} \cup\{4 k+2\}$.

Suppose $\left|a_{j}-a_{j+1}\right|=r$. We may choose the circular permutation starting at $a_{j+1}$ and ending at $a_{j}$. This is a required sequence.

Actually, these labelings induce local antimagic 3-labelings of cycles accordingly.

Adding each term of the circular permutation above by $a \in \mathbb{N}$, we have

Corollary 3.2. Suppose $N \geq 2, a \in \mathbb{N}$ and

$$
r \in \begin{cases}\{1\}, & N=2 ; \\ ([2, N-2] \cap \mathbb{E}) \cup\{1, N-1\}, & \text { even } N \geq 4 ; \\ ([1, N-2] \cap \mathbb{O}) \cup\{N-1\}, & \text { odd } N,\end{cases}
$$

where $\mathbb{E}$ and $\mathbb{O}$ are the set of even and odd integers, respectively. All integers in $[a+1, a+N]$ can be arranged as a sequence $\left(a_{i}\right)_{i=1}^{N}$ of length $N$ such that the sum of two consecutive terms $a_{i}+a_{i+1} \in\{2 a+N, 2 a+N+$ $1,2 a+N+2\}$ for $1 \leq i \leq N-1$ and $\left|a_{N}-a_{1}\right|=r$.

In this section, we consider $S p\left(n_{1}, n_{2}, n_{3}\right)$, spider graphs with 3 legs. Let its three legs be $P_{n_{1}+1}=$ $x_{1} \cdots x_{n_{1}} x_{n_{1}+1}, P_{n_{2}+1}=y_{1} \cdots y_{n_{2}} y_{n_{2}+1}$ and $P_{n_{3}+1}=z_{1} \cdots z_{n_{3}} z_{n_{3}+1}$, where $x_{n_{1}+1}=y_{n_{2}+1}=z_{n_{3}+1}=u$.

Let $P=x_{1} \cdots x_{n}$ and $Q=y_{1} \cdots y_{m}, n, m \geq 2$. The graph $P Q$ is the path obtained from $P$ and $Q$ by identifying $x_{n}$ with $y_{1}$.

Lemma 3.3. Let $P_{n+1}=x_{1} \cdots x_{n+1}$ be a path of length $n \geq 2$. Let $a \notin\{0,1\}$. There is an edge labeling $f: E\left(P_{n+1}\right) \rightarrow[a, a+n-1]$ such that $f^{+}$is a coloring of $P_{n+1}$ and $f^{+}\left(x_{1}\right)=a+n-2, f^{+}\left(x_{n+1}\right)=a+n-1$ and $f^{+}\left(x_{i}\right) \in\{2 a+n-1,2 a+n-2,2 a+n-3\}$ for $2 \leq i \leq n$. Moreover, $f^{+}\left(x_{2}\right)=2 a+n-1=f^{+}\left(x_{n}\right)$. 
Proof. Suppose $n=2 k$ for some $k \geq 1$. Label the edges by

\begin{tabular}{c||c|c|c|c|c|c|c|c|c|}
$i$ & 1 & 2 & 3 & 4 & $\cdots$ & $2 k-3$ & $2 k-2$ & $2 k-1$ & $2 k$ \\
\hline \hline$x_{i} x_{i+1}$ & $a+2 k-2$ & $a+1$ & $a+2 k-4$ & $a+3$ & $\cdots$ & $a+2$ & $a+2 k-3$ & $a$ & $a+2 k-1$ \\
\hline
\end{tabular}

Precisely,

$$
f\left(x_{i} x_{i+1}\right)= \begin{cases}a+2 k-i-1 & \text { if } i \text { is odd; } \\ a+i-1 & \text { if } i \text { is even. }\end{cases}
$$

It is easy to see that $f^{+}\left(x_{1}\right)=a+2 k-2, f^{+}\left(x_{2 k+1}\right)=a+2 k-1, f^{+}\left(x_{i}\right)=2 a+2 k-1$ for even $i$, $f^{+}\left(x_{i}\right)=2 a+2 k-3$ for odd $i \in[3,2 k-1]$. Clearly $f^{+}$is a 4 -coloring.

Suppose $n=4 k+1$ for some $k \geq 1$. Label the edges by

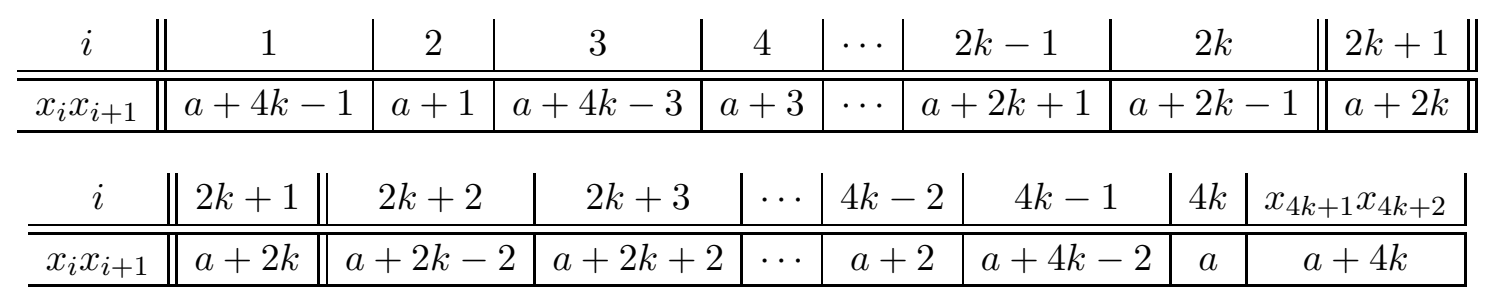

Precisely,

$$
f\left(x_{i} x_{i+1}\right)= \begin{cases}a+4 k-i & \text { if } i \text { is odd and } 1 \leq i \leq 2 k-1 \\ a+2 k & \text { if } i=2 k+1 \\ a+i-1 & \text { if } i \text { is odd and } 2 k+3 \leq i \leq 4 k+1 \\ a+i-1 & \text { if } i \text { is even and } 2 \leq i \leq 2 k ; \\ a+4 k-i & \text { if } i \text { is even and } 2 k+2 \leq i \leq 4 k .\end{cases}
$$

It is easy to see that $f^{+}\left(x_{1}\right)=a+4 k-1, f^{+}\left(x_{4 k+2}\right)=a+4 k, f^{+}\left(x_{i}\right)=2 a+4 k$ for even $i \in[2,2 k]$, $f^{+}\left(x_{i}\right)=2 a+4 k-2$ for even $i \in[2 k+2,4 k], f^{+}\left(x_{i}\right)=2 a+4 k-2$ for odd $i \in[3,2 k-1], f^{+}\left(x_{2 k+1}\right)=2 a+4 k-1$, $f^{+}\left(x_{i}\right)=2 a+4 k$ for odd $i \in[2 k+3,4 k+1]$. Clearly $f^{+}$is a 5 -coloring.

Suppose $n=4 k+3$ for some $k \geq 0$. Label the edges by

\begin{tabular}{c||c|c|c|c|c|c|c|c||c||}
$i$ & 1 & 2 & 3 & 4 & $\cdots$ & $2 k-1$ & $2 k$ & $2 k+1$ & $2 k+2$ \\
\hline \hline$x_{i} x_{i+1}$ & $a+4 k+1$ & $a+1$ & $a+4 k-1$ & $a+3$ & $\cdots$ & $a+2 k+3$ & $a+2 k-1$ & $a+2 k+1$ & $a+2 k$ \\
\hline
\end{tabular}

\begin{tabular}{c||c||c|c|c|c|c|c|c|}
$i$ & $2 k+2$ & $2 k+3$ & $2 k+4$ & $\cdots$ & $4 k$ & $4 k+1$ & $4 k+2$ & $4 k+3$ \\
\hline \hline$x_{i} x_{i+1}$ & $a+2 k$ & $a+2 k+2$ & $a+2 k-2$ & $\cdots$ & $a+2$ & $a+4 k$ & $a$ & $a+4 k+2$ \\
\hline
\end{tabular}

Precisely,

$$
f\left(x_{i} x_{i+1}\right)= \begin{cases}a+4 k+2-i & \text { if } i \text { is odd and } 1 \leq i \leq 2 k+1 \\ a+i-1 & \text { if } i \text { is odd and } 2 k+3 \leq i \leq 4 k+3 \\ a+i-1 & \text { if } i \text { is even and } 2 \leq i \leq 2 k \\ a+4 k+2-i & \text { if } i \text { is even and } 2 k+2 \leq i \leq 4 k+2 .\end{cases}
$$

It is easy to see that $f^{+}\left(x_{1}\right)=a+4 k+1, f^{+}\left(x_{4 k+4}\right)=a+4 k+2, f^{+}\left(x_{i}\right)=2 a+4 k+2$ for even $i \in[2,2 k]$, $f^{+}\left(x_{2 k+2}\right)=2 a+4 k+1, f^{+}\left(x_{i}\right)=2 a+4 k$ for even $i \in[2 k+4,4 k+2], f^{+}\left(x_{i}\right)=2 a+4 k$ for odd $i \in[3,2 k+1]$, $f^{+}\left(x_{i}\right)=2 a+4 k+2$ for odd $i \in[2 k+3,4 k+3]$. Clearly $f^{+}$is a 5 -coloring.

The last statement is easy to check. 
Theorem 3.4. $\chi_{l a}(S p(2,2+2 m, 2+2 m+k))=4$ for $m \geq 0$ and $k \geq 2$.

Proof. Let $f$ be the required labeling. Now $q=6+4 m+k$. Let $P_{3}^{(1)}=x_{1} x_{2} x_{3}, P_{3}^{(2)}=y_{1} y_{2} y_{3}$ and $P_{3}^{(3)}=z_{1} z_{2} z_{3}, Q_{2 m+1}^{(1)}=v_{1} \cdots v_{2 m} v_{2 m+1}, Q_{2 m+1}^{(2)}=w_{1} \cdots w_{2 m} w_{2 m+1}$, and $R_{k+1}=u_{1} \cdots u_{k} u_{k+1}$.

We label $P_{3}^{(j)}$ by $f\left(x_{1} x_{2}\right)=q, f\left(x_{2} x_{3}\right)=1, f\left(y_{1} y_{2}\right)=q-1, f\left(y_{2} y_{3}\right)=2, f\left(z_{1} z_{2}\right)=q-2, f\left(z_{2} z_{3}\right)=3$ and $Q_{2 m+1}^{(l)}, 1 \leq l \leq 2$, by

\begin{tabular}{c||c|c|c|c|c|c|c|}
$i$ & 1 & 2 & 3 & 4 & $\cdots$ & $2 m-1$ & $2 m$ \\
\hline \hline$v_{i} v_{i+1}$ & $q-3 n$ & $3 n+1$ & $q-3 n-2$ & $3 n+3$ & $\cdots$ & $q-3 n-2 m+2$ & $3 n+2 m-1$ \\
\hline$w_{i} w_{i+1}$ & $q-3 n-1$ & $3 n+2$ & $q-3 n-3$ & $3 n+4$ & $\cdots$ & $q-3 n-2 m+1$ & $3 n+2 m$ \\
\hline
\end{tabular}

The vertex labels of $v_{1} \cdots v_{2 m}$ and $w_{1} \cdots w_{2 m}$ are $q+1$ and $q-1$ alternatively.

Let $P_{3}=P_{3}^{(1)}, P_{3+2 m}=P_{3}^{(2)} Q_{2 m+1}^{(1)}$ and $P_{3+2 m+k}=P_{3}^{(3)} Q_{2 m+1}^{(2)} R_{k+1}$. By Lemma 3.3, we may label $R_{k+1}$ by $[4+2 m, 3+2 m+k]$ such that $f^{+}\left(u_{k+1}\right)=3+2 m+k, f^{+}\left(u_{1}\right)=2+2 m+k$ and $f^{+}\left(u_{2}\right)=$ $7+4 m+k=f^{+}\left(u_{k}\right)$. Let the combined labeling still be denoted by $f$. Then $f^{+}\left(z_{3}\right)=f^{+}\left(y_{3}\right)=q-1$, $f^{+}\left(w_{2 m+1}\right)=(3+2 m)+(2+2 m+k)=5+4 m+k$ and $f^{+}(u)=1+(2+2 m)+(3+2 m+k)=6+4 m+k$.

Theorem 3.5. Suppose $n \geq 1, m \geq 0$ and $l \geq m+2$. $\chi_{l a}(\operatorname{Sp}(2 n, 2 n+2 m+1, l))=4$.

Proof. Here the number of edges is $q=4 n+2 m+l+1$. Let $P_{2 n+1}^{(1)}=x_{1} \cdots x_{2 n} x_{2 n+1}, P_{2 n+1}^{(2)}=y_{1} \cdots y_{2 n+1}$ and $Q_{2 m+2}=v_{1} \cdots v_{2 m} v_{2 m+2}$. Also, let the last leg of the spider be $P_{l+1}=z_{1} \cdots z_{l+1}$. Let $f$ be a required labeling. Firstly, let $f\left(z_{1} z_{2}\right)=q, f\left(z_{2} z_{3}\right)=x$ and $f\left(z_{l} z_{l+1}\right)=y$.

We label $P_{2 n+1}^{(j)}$ and $Q_{2 m+2}, 1 \leq j \leq 2$, by

\begin{tabular}{c||c|c|c|c|c|c|c|}
$i$ & 1 & 2 & 3 & 4 & $\cdots$ & $2 n-1$ & $2 n$ \\
\hline \hline$x_{i} x_{i+1}$ & $q-1$ & 1 & $q-3$ & 3 & $\cdots$ & $q-2 n+1$ & $2 n-1$ \\
\hline$y_{i} y_{i+1}$ & $q-2$ & 2 & $q-4$ & 4 & $\cdots$ & $q-2 n$ & $2 n$ \\
\hline
\end{tabular}

\begin{tabular}{c||c|c|c|c|c|c|c|c|c|}
$i$ & 1 & 2 & 3 & 4 & $\cdots$ & $2 m-2$ & $2 m-1$ & $2 m$ & $2 m+1$ \\
\hline \hline$v_{i} v_{i+1}$ & $q-2 n-1$ & $2 n+1$ & $q-2 n-2$ & $2 n+2$ & $\cdots$ & $2 n+m-1$ & $q-2 n-m$ & $2 n+m$ & $q-2 n-m-1$ \\
\hline
\end{tabular}

Following we shall label all $l-1$ unlabeled edges of the path $P_{l+1}=z_{1} \cdots z_{l+1}$ by integers in $[2 n+m+1,2 n+m+l-1]=[a+1, a+N]$, where $a=2 n+m$ and $N=l-1$.

(a) Suppose $l \equiv m(\bmod 2)$ and $l \geq m+4$. Let the first two legs of the spider be $P_{2 n+1}=P_{2 n+1}^{(2)}$ and $P_{2 n+2 m+2}=P_{2 n+1}^{(1)} Q_{2 m+2}$. We require $q+x=f^{+}\left(z_{2}\right)=f^{+}(u)=f\left(z_{l} z_{l+1}\right)+(2 n)+(q-2 n-m-1)=$ $y+q-m-1$ and $f^{+}\left(z_{i}\right) \in\{q-2, q-1, q\}$ for $3 \leq i \leq l$. It is equivalent to $x+m+1=y$.

Let $r=m+1$. Then $N=l-1>r$ and $r \equiv N(\bmod 2)$. By Corollary 3.2 with this $r, N$ and $a$, we can label $z_{2} \cdots z_{l+1}$ satisfying the above requirement. Note that $2 a+N=4 n+2 m+l-1$. Hence $f$ is a local antimagic 4-labeling for $S p(2 n, 2 n+2 m+1, l)$.

(b) Suppose $l \not \equiv m(\bmod 2)$ and $l \geq m+4$. Let the first two legs of the spider be $P_{2 n+1}=P_{2 n+1}^{(1)}$ and $P_{2 n+2 m+2}=P_{2 n+1}^{(2)} Q_{2 m+2}$. Similarly, we require $x+m+2=y$. Let $r=m+2$. Now $N>r$ and $N \equiv r$ $(\bmod 2)$. By Corollary 3.2 , we can label $z_{2} \cdots z_{l+1}$ satisfying the above requirement. Hence $f$ is a local antimagic 4-labeling for $S p(2 n, 2 n+2 m+1, l)$.

(c) Suppose $l=m+3$. We use the same labeling as the case (a). We require that $x+m+1=y$. Let $r=m+1$. Then $N=m+2$, i.e., $r=N-1$. By Corollary 3.2, we have a local antimagic 4-labeling for $S p(2 n, 2 n+2 m+1, m+3)$. 
(d) Suppose $l=m+2$.

(i) Suppose $m \geq 1$. We use the labeling defined in the case (a), but swap the labels of the edges $v_{2 m-1} v_{2 m}$ and $v_{2 m+1} v_{2 m+2}$. Now, we require that $x+m=y$. Let $r=m$. Then $r=N-1$. By Corollary 3.2 , we have a local antimagic 4-labeling for $S p(2 n, 2 n+2 m+1, m+2)$.

(ii) Suppose $m=0$. If $n \geq 2$, then $S p(2 n, 2 n+1,2)=S p(2,2+2(n-1)+1,2 n)$. Since $2 n \geq n+2$, by the cases (a), (b) or (c) we get the result. If $n=1$, then $S p(2,3,2)=S p(2,2,3)$. A required labeling is 7,$2 ; 6,3 ; 5,4,1$ with induced colors $9,7,6,5$.

Theorem 3.6. Suppose $n \geq 1, m \geq 0$ and $l \geq 2$. $\chi_{l a}(S p(2 n+1,2 n+2 m, l))=4$.

Proof. When $m=0 . S p(2 n+1,2 n+2 m, l)=S p(2 n, 2 n+1, l)$. By Theorem 3.5 we get $\chi_{l a}(S p(2 n+1,2 n+$ $2 m, l))=4$. Following we assume $m \geq 1$.

Suppose $l=2 . S p(2 n+1,2 n+2 m, 2)=S p(2,2(n-1)+3,2 n+2 m)$. Clearly, $2 n+2 m>(n-1)+3$.

By Theorem 3.5 (with $n=1)$ we have $\chi_{l a}(S p(2,2(n-1)+3,2 n+2 m))=4$.

So we assume $l \geq 3$.

A. Suppose $l \geq m$. Let $P_{2 n+2}^{(1)}=x_{1} \cdots x_{2 n} x_{2 n+2}, P_{2 n+2}^{(2)}=y_{1} \cdots y_{2 n+2}$ and $Q_{2 m}=v_{1} \cdots v_{2 m}$. Also, let the last leg of the spider be $P_{l+1}=z_{1} \cdots z_{l+1}$. Now the number of edges is $q=4 n+2 m+l+1$.

Let $f$ be a required labeling. Firstly, let $f\left(z_{1} z_{2}\right)=q, f\left(z_{2} z_{3}\right)=x$ and $f\left(z_{l} z_{l+1}\right)=y$.

We label $P_{2 n+2}^{(j)}$ and $Q_{2 m}, 1 \leq j \leq 2$, by

\begin{tabular}{c||c|c|c|c|c|c|c|c|}
$i$ & 1 & 2 & 3 & 4 & $\cdots$ & $2 n-1$ & $2 n$ & $2 n+1$ \\
\hline \hline$x_{i} x_{i+1}$ & $q-1$ & 1 & $q-3$ & 3 & $\cdots$ & $q-2 n+1$ & $2 n-1$ & $q-2 n-1$ \\
\hline$y_{i} y_{i+1}$ & $q-2$ & 2 & $q-4$ & 4 & $\cdots$ & $q-2 n$ & $2 n$ & $q-2 n-2$ \\
\hline
\end{tabular}

\begin{tabular}{c||c|c|c|c|c|c|c|}
$i$ & 1 & 2 & 3 & $\cdots$ & $2 m-3$ & $2 m-2$ & $2 m-1$ \\
\hline \hline$v_{i} v_{i+1}$ & $2 n+1$ & $q-2 n-3$ & $2 n+2$ & $\cdots$ & $2 n+m-1$ & $q-2 n-m-1$ & $2 n+m$ \\
\hline
\end{tabular}

Similar to the proof of Theorem 3.5, we shall label all $l-1$ unlabeled edges of the path $P_{l+1}=z_{1} \cdots z_{l+1}$ by integers in $[a+1, a+N]$, where $a=2 n+m$ and $N=l-1$.

We assume $m \geq 3$ first.

(a) Suppose $l \equiv m(\bmod 2)$ and $l>m$. Let the first two legs of the spider be $P_{2 n+2}=P_{2 n+2}^{(1)}$ and $P_{2 n+2 m+1}=P_{2 n+2}^{(2)} Q_{2 m}$. Similar to the proof of Theorem 3.5 we require $q+x=f^{+}\left(z_{2}\right)=f^{+}(u)=$ $f\left(z_{l} z_{l+1}\right)+(q-2 n-1)+(2 n+m)=y+q+m-1$. It is equivalent to $x=y+m-1$. Let $r=m-1$ and $N=l-1$. By Corollary 3.2 , we have the result.

(b) Suppose $l \not \equiv m(\bmod 2)$. Let the first two legs of the spider be $P_{2 n+2}=P_{2 n+2}^{(2)}$ and $P_{2 n+2 m+1}=$ $P_{2 n+2}^{(1)} Q_{2 m}$. Similarly, we require $x=y+m-2$. Let $r=m-2$ and $N=l-1$. By Corollary 3.2 , we have the result.

(c) Suppose $l=m$. We use the labeling defined in (b). Now $r=m-2=N-1$. By Corollary [3.2, we have the result.

When $m=1$. Use the labeling of case (b). We require $x=y-1$. That is, $r=1$ and $N=l-1 \geq 2$. By Corollary [3.2, $f$ is a local antimagic 4-labeling for $S p(2 n+1,2 n+2 m, l)$.

When $m=2$. Use the labeling of case (a). We require $x=y+1$. By Corollary 3.2, $f$ is a local antimagic 4-labeling for $S p(2 n+1,2 n+2 m, l)$. 
B. Suppose $l<m$.

(a) Suppose $l=2 h \leq 2 n$. Then $S p(2 n+1,2 n+2 m, l)=S p(2 h, 2 n+1,2 n+2 m)=S p(2 h, 2 h+2(n-$ $h)+1,2 n+2 m)$. Clearly, $2 n+2 m \geq(n-h)+3$. By Theorem 3.5, $\chi_{l a}(S p(2 n+1,2 n+2 m, l))=4$.

(b) Suppose $l=2 n+2 s$, where $s \geq 1 . S p(2 n+1,2 n+2 m, l)=S p(2 n+1,2 n+2 m, 2 n+2 s)=S p(2 n+$ $1,2 n+2 s, 2 n+2 m)$. Clearly, $2 n+2 m \geq \max \{3, s\}$. By the Case A, $\chi_{l a}(S p(2 n+1,2 n+2 m, l))=4$.

(c) Suppose $l=2 h+1$. Since $m \geq 4$, we let $m=2 h+k, k \geq 2$ to get $S p(2 n+1,2 h+1,2 n+4 h+2 k)$. Now, $q=4 n+6 h+2 k+2$. Let $P_{2 n+2}^{(1)}=x_{1} \cdots x_{2 n+1} x_{2 n+2}, P_{2 n+2}^{(2)}=y_{1} \cdots y_{2 n+1} y_{2 n+2}, Q_{4 h+1}=z_{1} \cdots z_{4 h+1}$, $S_{2 k}=w_{1} \cdots w_{2 k}$ and $R_{2 h+2}=v_{1} \cdots v_{2 h+1} v_{2 h+2}$. Let $f$ be a required labeling. Firstly, let $f\left(v_{1} v_{2}\right)=q$, $f\left(v_{2} v_{3}\right)=x$ and $f\left(v_{2 h+1} z_{2 h+2}\right)=y$.

We label $P_{2 n+2}^{(j)}$ by integers in $[1,2 n] \cup[q-2 n-2, q-1]$ :

\begin{tabular}{c||c|c|c|c|c|c|c|c|}
$i$ & 1 & 2 & 3 & 4 & $\cdots$ & $2 n-1$ & $2 n$ & $2 n+1$ \\
\hline \hline$x_{i} x_{i+1}$ & $q-1$ & 1 & $q-3$ & 3 & $\cdots$ & $q-2 n+1$ & $2 n-1$ & $q-2 n-1$ \\
\hline$y_{i} y_{i+1}$ & $q-2$ & 2 & $q-4$ & 4 & $\cdots$ & $q-2 n$ & $2 n$ & $q-2 n-2$ \\
\hline
\end{tabular}

We label $Q_{4 h+1}$ by in integers in $[2 n+1,2 n+2 h] \cup[2 n+4 h+2 k, 2 n+6 h+2 k-1=q-2 n-3]$ :

\begin{tabular}{c||c|c|c|c|c|c|c|c|c|}
$i$ & 1 & 2 & 3 & 4 & $\cdots$ & $4 h-3$ & $4 h-2$ & $4 h-1$ & $4 h$ \\
\hline \hline$z_{i} z_{i+1}$ & $2 n+1$ & $q-2 n-3$ & $2 n+2$ & $q-2 n-4$ & $\cdots$ & $2 n+2 h-1$ & $2 n+4 h+2 k+1$ & $2 n+2 h$ & $2 n+4 h+2 k$ \\
\hline
\end{tabular}

We label $S_{2 k}$ by integers in $[2 n+2 h+1,2 n+2 h+k] \cup[2 n+4 h+k+1,2 n+4 h+2 k-1]$. If $k$ is even, we have

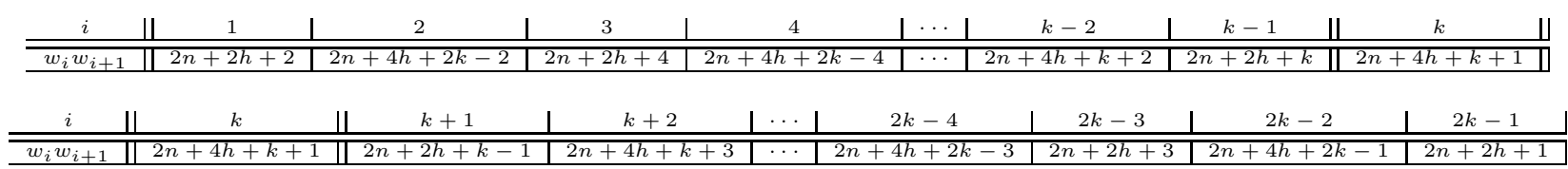

If $k$ is odd, we have

\begin{tabular}{c||c|c|c|c|c|c|c|c|c|}
$i$ & 1 & 2 & 3 & 4 & $k-2$ & $k-1$ \\
\hline \hline$w_{i} w_{i+1}$ & $2 n+2 h+2$ & $2 n+4 h+2 k-2$ & $2 n+2 h+4$ & $2 n+4 h+2 k-4$ & $\cdots$ & $2 n+2 h+k-1$ & $2 n+4 h+k+1$ & $2 n+2 h+k$ \\
\hline & \\
$i$ & $k$ & $k+1$ & $k+2$ & $\cdots$ & $2 k-4$ & $2 k-3$ & $2 k-2$ \\
\hline \hline$w_{i} w_{i+1}$ & $2 n+2 h+k$ & $2 n+4 h+k+2$ & $2 n+2 h+k-2$ & $\cdots$ & $2 n+4 h+2 k-3$ & $2 n+2 h+3$ & $2 n+4 h+2 k-1$ & $2 n+2 h+1$ \\
\hline
\end{tabular}

We now have $P_{2 n+4 h+2 k+1}=P_{2 n+2}^{(1)} Q_{4 h+1} S_{2 k}$. Moreover, $P_{2 n+2}=P_{2 n+2}^{(2)}$. We shall now label the remaining $2 h$ edge of $R_{2 h+2}$ using integers in $[2 n+2 h+k+1,2 n+4 h+k]$. Similar to the proof of the case A, we require $x=y+2 h-1$. That is, $r=2 h-1$ and $N=2 h$. By Corollary 3.2 , we have a local antimagic 4-labeling for $S p(2 n+1,2 h+1,2 n+4 h+2 k)$.

Theorem 3.7. For $n \geq 1$ and $2 \leq l \leq m+1, \chi_{l a}(\operatorname{Sp}(2 n, 2 n+2 m+1, l))=4$.

Proof.

(a) Suppose $l=2 h+1$ with $h<n$. Then $S p(2 n, 2 n+2 m+1,2 h+1)=S p(2 h+1,2 n, 2 n+2 m+1)=$ $S p(2 h+1,2 h+2(n-h), 2 n+2 m+1)$. Clearly, $2 n+2 m+1 \geq \max \{n-h, 3\}$. By Theorem 3.6. $\chi_{l a}(S p(2 n+1,2 n+2 m, l))=4$. 
(b) Suppose $l=2 h+1$ with $h \geq n$. Then $S p(2 n, 2 n+2 m+1,2 h+1)=S p(2 n, 2 h+1,2 n+2 m+1)=$ $S p(2 n, 2 n+2(h-n)+1,2 n+2 m+1)$. Since $2 h+1 \leq m+1,2 n+2 m+1 \geq(h-n)+2$. By Theorem 3.5 , $\chi_{l a}(S p(2 n+1,2 n+2 m, l))=4$.

(c) Suppose $l=2 h, h \geq 1$. Since $l \leq m+1$, we let $m=2 h-1+2 k, k \geq 0$, to get $S p(2 n, 2 h, 2 n+4 h+4 k-1)$. Now, $q=4 n+6 h+4 k-1$.

Suppose $h \geq 3$. Similar to the proof of Theorem 3.5, let $P_{2 n+1}^{(1)}=x_{1} \cdots x_{2 n} x_{2 n+1}, P_{2 n+1}^{(2)}=y_{1} \cdots y_{2 n+1}$, $Q_{4 h+4 k}=v_{1} \cdots v_{2 h+4 k-1} v_{4 h+4 k}$ and $P_{2 h+1}=z_{1} \cdots z_{2 h+1}$. Let $f$ be a required labeling. Firstly, let $f\left(z_{1} z_{2}\right)=q, f\left(z_{2} z_{3}\right)=x$ and $f\left(z_{l} z_{l+1}\right)=y$.

We label $P_{2 n+1}^{(j)}, 1 \leq j \leq 2$, by

\begin{tabular}{c||c|c|c|c|c|c|c|}
$i$ & 1 & 2 & 3 & 4 & $\cdots$ & $2 n-1$ & $2 n$ \\
\hline \hline$x_{i} x_{i+1}$ & $q-1$ & 1 & $q-3$ & 3 & $\cdots$ & $q-2 n+1$ & $2 n-1$ \\
\hline$y_{i} y_{i+1}$ & $q-2$ & 2 & $q-4$ & 4 & $\cdots$ & $q-2 n$ & $2 n$ \\
\hline
\end{tabular}

We label $Q_{4 h+4 k}$ by

\begin{tabular}{c||c|c|c|c|c|c|c|c|c|}
$i$ & 1 & 2 & 3 & 4 & $\cdots$ & $2 h+2 k-3$ & $2 h+2 k-2$ & $2 h+2 k-1$ & $2 h+2 k$ \\
\hline \hline$v_{i} v_{i+1}$ & $q-2 n-1$ & $2 n+1$ & $q-2 n-3$ & $2 n+3$ & $\cdots$ & $2 n+4 h+2 k+2$ & $2 n+2 h+2 k-3$ & $2 n+4 h+2 k$ & $2 n+2 h+2 k-1$ \\
\hline
\end{tabular}

\begin{tabular}{c||c|c|c|c|c|c|c|c|c|c|c|c|}
$i$ & $2 h+2 k+1$ & $2 h+2 k+2$ & $2 h+2 k+3$ & $2 h+2 k+4$ & $\cdots$ & $4 h+4 k-3$ & $4 h+4 k-2$ & $4 h+4 k-1$ \\
\hline \hline$v_{i} v_{i+1}$ & $2 n+4 h+2 k-1$ & $2 n+2 h+2 k-2$ & $2 n+4 h+2 k+1$ & $2 n+2 h+2 k-4$ & $\cdots$ & $q-2 n-4$ & $2 n+2$ & $q-2 n-2$ \\
\hline
\end{tabular}

Let $P_{2 n+1}=P_{2 n+1}^{(1)}$ and $P_{2 n+4 h+4 k}=P_{2 n+1}^{(2)} Q_{4 h+4 k}$. We require $q+x=y+(2 n-1)+(q-2 n-2)=y+q-3$ so that $x+3=y$. Similar to the proof of Theorem [3.4, we require $r=3, a=2 n+2 h+2 k-1$ and $N=2 h-1 \geq 5$. By Corollary 3.2, we have a local antimagic 4-labeling for $S p(2 n, 2 h, 2 n+4 h+4 k-1)$. Suppose $h=1$ or $n=1$. By Theorem 3.4, $\chi_{l a}(S p(2 n, 2 h, 2 n+4 h+4 k-1))=4$.

Suppose $h=2$ and $n \geq 3$. We get $S p(4,2 n, 2 n+4 k+7)$. Let $P_{5}^{(1)}=x_{1} \cdots x_{5}, P_{5}^{(2)}=y_{1} \cdots y_{5}$, $Q_{2 n+4 k+4}=v_{1} \cdots v_{2 n+4 k+4}$ and $P_{2 n+1}=z_{1} \cdots z_{2 n+1}$. Label the edges according to the steps above, we can get a similar conclusion.

If $n=h=2$, we have $S p(4,4,4 k+11), k \geq 0$. Let $P_{5}^{(1)}=x_{1} x_{2} \cdots x_{5}, P_{5}^{(2)}=y_{1} y_{2} \cdots y_{5}$ and $P_{5}^{(3)}=$ $z_{1} z_{2} \cdots z_{5}$. Also let $Q_{4 k+8}=v_{1} v_{2} \cdots v_{4 k+8}$. We label $P_{5}^{(j)}, j=1,2,3$ by

\begin{tabular}{c||c|c|c|c|}
$i$ & 1 & 2 & 3 & 4 \\
\hline \hline$x_{i} x_{i+1}$ & $4 k+18$ & 1 & $4 k+16$ & 3 \\
\hline$y_{i} y_{i+1}$ & $4 k+17$ & 2 & $4 k+15$ & 4 \\
\hline$z_{i} z_{i+1}$ & $4 k+19$ & $2 k+8$ & $2 k+9$ & $2 k+10$ \\
\hline
\end{tabular}

Label $Q_{4 k+8}$ using integers in $[5,2 k+7] \cup[2 k+11,4 k+14]$ by

\begin{tabular}{c||c|c|c|c|c|c|c|c|c}
$i$ & 1 & 2 & 3 & 4 & $\cdots$ & $2 k+1$ & $2 k+2$ & $2 k+3$ & $2 k+4$ \\
\hline \hline$v_{i} v_{i+1}$ & $4 k+14$ & 5 & $4 k+12$ & 7 & $\cdots$ & $2 k+14$ & $2 k+5$ & $2 k+12$ & $2 k+7$ \\
\hline \multicolumn{10}{c}{} \\
$i$ & $2 k+5$ & $2 k+6$ & $2 k+7$ & $2 k+8$ & $\cdots$ & $4 k+5$ & $4 k+6$ & $4 k+7$ \\
\hline \hline$v_{i} v_{i+1}$ & $2 k+11$ & $2 k+6$ & $2 k+13$ & $2 k+4$ & $\cdots$ & $4 k+11$ & 6 & $4 k+13$ \\
\hline
\end{tabular}

Let $P_{4 k+12}=P_{5}^{(1)} Q_{4 k+8}$ and the two paths of length 5 be $P_{5}^{(j)}, j=2,3$. It is easy to check that $S p(4,4,4 k+11)$ admits a local antimagic 4 -labeling with vertex labels in $\{4 k+17,4 k+18,4 k+19,6 k+27\}$. 
Combining Theorems 3.5, 3.6 and 3.7, we have

Theorem 3.8. For $a, b, c \geq 2$ with even $a$ and odd $b, \chi_{l a}(S p(a, b, c))=4$.

\section{Spider with three even legs}

Now we are going to consider the case for three even legs.

Theorem 4.1. For $h \geq m \geq n \geq 1, \chi_{l a}(S p(2 n, 2 m, 2 h))=4$.

Proof. By Corollary 2.10 and Theorem 3.4, we may assume $h>m>n>1$. Let $f: E(S p(2 n, 2 m, 2 h)) \rightarrow$ $[1,2 n+2 m+2 h]$ be a bijection. Note that $q=2 n+2 m+2 h$. Let $r=q / 2=n+m+h$. Let $P_{2 n+1}=$ $x_{1} x_{2} \cdots x_{2 n+1}, P_{2 m+1}=y_{1} y_{2} \cdots y_{2 m+1}, P_{2 h+1}=z_{1} z_{2} \cdots z_{2 h+1}$. We first consider $(m, h) \neq(n+1, n+2)$. Let $f\left(x_{1} x_{2}\right)=q-1, f\left(y_{1} y_{2}\right)=q-2$ and $f\left(z_{1} z_{2}\right)=q$.

Label the remaining edges of $P_{2 n+1}$ as:

\begin{tabular}{c||c|c|c|c|c|c|c|c|c|}
$i$ & 2 & 3 & 4 & 5 & $\cdots$ & $2 n-3$ & $2 n-2$ & $2 n-1$ & $2 n$ \\
\hline \hline$x_{i} x_{i+1}$ & $r$ & $r-2$ & $r+2$ & $r-4$ & $\cdots$ & $r-2 n+4$ & $r+2 n-4$ & $r-2 n+2$ & $r+2 n-2$ \\
\hline
\end{tabular}

For $P_{2 h+1}$, we label the subpath $z_{2} z_{3} \cdots z_{2 n+2}$ as:

\begin{tabular}{c||c|c|c|c|c|c|c|c|c|}
$k$ & 2 & 3 & 4 & 5 & $\cdots$ & $2 n-2$ & $2 n-1$ & $2 n$ & $2 n+1$ \\
\hline \hline$z_{k} z_{k+1}$ & $r-1$ & $r+1$ & $r-3$ & $r+3$ & $\cdots$ & $r-2 n+3$ & $r+2 n-3$ & $r-2 n+1$ & $r+2 n-1$ \\
\hline
\end{tabular}

For odd $m$, label the remaining edges of $P_{2 m+1}$ as:

\begin{tabular}{c||c|c|c|c|c|c|c|c|c|c|}
$j$ & 2 & 3 & 4 & 5 & $\cdots$ & $m-3$ & $m-2$ & $m-1$ & $m$ & $m+1$ \\
\hline \hline$y_{i} y_{j+1}$ & 1 & $q-3$ & 3 & $q-5$ & $\cdots$ & $m-4$ & $q-m+2$ & $m-2$ & $q-m$ & $m$ \\
\hline$y_{m+j} y_{m+1+j}$ & $q-m-1$ & $m-1$ & $q-m+1$ & $m-3$ & $\cdots$ & $q-6$ & 4 & $q-4$ & 2 & \\
\hline
\end{tabular}

For even $m$, label the remaining edges of $P_{2 m+1}$ as:

\begin{tabular}{c||c|c|c|c|c|c|c|c|c|c|}
$j$ & 2 & 3 & 4 & 5 & $\cdots$ & $m-3$ & $m-2$ & $m-1$ & $m$ & $m+1$ \\
\hline \hline$y_{i} y_{j+1}$ & 1 & $q-3$ & 3 & $q-5$ & $\cdots$ & $q-m+3$ & $m-3$ & $q-m+1$ & $m-1$ & $q-m-1$ \\
\hline$y_{m+j} y_{m+1+j}$ & $m$ & $q-m$ & $m-2$ & $q-m+2$ & $\cdots$ & $q-6$ & 4 & $q-4$ & 2 & \\
\hline
\end{tabular}

Up to now, $[1, m] \cup[r-2 n+1, r+2 n-1] \cup[q-m-1, q]$ are used. Note that, $r-2 n+1 \geq m+2$ and $r+2 n-1 \leq 2 n+m+2 h-3=q-m-3$. So no label is used twice. Now we shall assign the labels in $[m+1, r-2 n] \cup[r+2 n, q-m-2]$ to the unlabeled edges of $P_{2 h+1}$.

For $n \equiv h(\bmod 2)$, we label the remaining edges of $P_{2 h+1}$ as:

\begin{tabular}{c||c|c|c|c|c|c|c|c|c|}
$k$ & $2 n+2$ & $2 n+3$ & $2 n+4$ & $2 n+5$ & $\cdots$ & $n+h-2$ & $n+h-1$ & $n+h$ & $n+h+1$ \\
\hline \hline$z_{k} z_{k+1}$ & $r-2 n$ & $r+2 n$ & $r-2 n-2$ & $r+2 n+2$ & $\cdots$ & $m+4$ & $q-m-4$ & $m+2$ & $q-m-2$ \\
\hline
\end{tabular}

\begin{tabular}{c||c|c|c|c|c|c|c|c|c|}
$k$ & 3 & 4 & 5 & 6 & $\cdots$ & $h-n-2$ & $h-n-1$ & $h-n$ & $h-n+1$ \\
\hline \hline$z_{n+h-1+k} z_{n+h+k}$ & $m+1$ & $q-m-3$ & $m+3$ & $q-m-5$ & $\cdots$ & $r+2 n+3$ & $r-2 n-3$ & $r+2 n+1$ & $r-2 n-1$ \\
\hline
\end{tabular}

For $n \not \equiv h(\bmod 2)$, we label the remaining edges of $P_{2 h+1}$ as:

\begin{tabular}{c||c|c|c|c|c|c|c|c|c|}
$k$ & $2 n+2$ & $2 n+3$ & $2 n+4$ & $2 n+5$ & $\cdots$ & $n+h-2$ & $n+h-1$ & $n+h$ & $n+h+1$ \\
\hline \hline$z_{k} z_{k+1}$ & $r-2 n$ & $r+2 n$ & $r-2 n-2$ & $r+2 n+4$ & $\cdots$ & $q-m-5$ & $m+3$ & $q-m-3$ & $m+1$ \\
\hline
\end{tabular}




\begin{tabular}{c||c|c|c|c|c|c|c|c|c|}
$k$ & 3 & 4 & 5 & 6 & $\cdots$ & $h-n-2$ & $h-n-1$ & $h-n$ & $h-n+1$ \\
\hline \hline$z_{n+h-1+k} z_{n+h+k}$ & $q-m-2$ & $m+2$ & $q-m-4$ & $m+4$ & $\cdots$ & $r+2 n+3$ & $r-2 n-3$ & $r+2 n+1$ & $r-2 n-1$ \\
\hline
\end{tabular}

Let $u=x_{2 n+1}=y_{2 m+1}=z_{2 h+1}$. When $(2 n, 2 m, 2 h) \neq(2 n, 2 n+2,2 n+4)$. It is routine to check that $f^{+}(u)=2 r-1=q-1, f^{+}\left(x_{2 n}\right)=f^{+}\left(z_{2 h}\right)=q, f^{+}\left(y_{2 m}\right)=q-2, f^{+}\left(x_{2}\right)=f^{+}\left(z_{2}\right)=q+r-1$, and all other induced vertex labels are $q-2, q-1$ and $q$. Thus, $f$ is a local antimagic 4-labeling.

Consider $S p(2 n, 2 n+2,2 n+4), n \geq 2$. Now $q=6 n+6$. Let $f\left(x_{1} x_{2}\right)=6 n+5, f\left(y_{1} y_{2}\right)=6 n+4$ and $f\left(z_{1} z_{2}\right)=6 n+6$. Label the remaining edges of $P_{2 n+1}$ as:

\begin{tabular}{c||c|c|c|c|c|c|c|c|c|}
$i$ & 2 & 3 & 4 & 5 & $\cdots$ & $2 n-3$ & $2 n-2$ & $2 n-1$ & $2 n$ \\
\hline \hline$x_{i} x_{i+1}$ & $3 n+3$ & $3 n+1$ & $3 n+5$ & $3 n-1$ & $\cdots$ & $n+7$ & $5 n-1$ & $n+5$ & $5 n+1$ \\
\hline
\end{tabular}

Label the remaining edges of $P_{2 n+5}$ as:

\begin{tabular}{c||c|c|c|c|c|c|c|c|c||c|c|}
$k$ & 2 & 3 & 4 & 5 & $\cdots$ & $2 n-1$ & $2 n$ & $2 n+1$ & $2 n+2$ & $2 n+3$ & $2 n+4$ \\
\hline \hline$z_{k} z_{k+1}$ & $3 n+2$ & $3 n+4$ & $3 n$ & $3 n+6$ & $\cdots$ & $5 n$ & $n+4$ & $5 n+2$ & $n+2$ & $5 n+3$ & $n+3$ \\
\hline
\end{tabular}

Now we used labels $[n+2,5 n+3] \cup\{6 n+4,6 n+5,6 n+6\}$.

For even $n$, label the remaining edges of $P_{2 n+3}$ as:

\begin{tabular}{c||c|c|c|c|c|c|c|c|c|}
$j$ & 2 & 3 & 4 & 5 & $\cdots$ & $n-1$ & $n$ & $n+1$ & $n+2$ \\
\hline \hline$y_{i} y_{j+1}$ & 2 & $6 n+2$ & 4 & $6 n$ & $\cdots$ & $5 n+6$ & $n$ & $5 n+4$ & \\
\hline$y_{n+j} y_{n+1+j}$ & $n+1$ & $5 n+5$ & $n-1$ & $5 n+7$ & $\cdots$ & $6 n+1$ & 3 & $6 n+3$ & 1 \\
\hline
\end{tabular}

For odd $n$, label the remaining edges of $P_{2 n+3}$ as:

\begin{tabular}{c||c|c|c|c|c|c|c|c|c|}
$j$ & 2 & 3 & 4 & 5 & $\cdots$ & $n-1$ & $n$ & $n+1$ & $n+2$ \\
\hline \hline$y_{i} y_{j+1}$ & 2 & $6 n+2$ & 4 & $6 n$ & $\cdots$ & $n-1$ & $5 n+5$ & $n+1$ & \\
\hline$y_{n+j} y_{n+1+j}$ & $5 n+4$ & $n$ & $5 n+6$ & $n-2$ & $\cdots$ & $6 n+1$ & 3 & $6 n+3$ & 1 \\
\hline
\end{tabular}

It is routine to check that $f^{+}(u)=6 n+5, f^{+}\left(x_{2 n}\right)=f^{+}\left(z_{2 n+4}\right)=6 n+6, f^{+}\left(y_{2 n+2}\right)=6 n+4$, $f^{+}\left(x_{2}\right)=f^{+}\left(z_{2}\right)=9 n+8$, and all other induced vertex labels are $6 n+4,6 n+5$ and $6 n+6$. Thus, $f$ is a local antimagic 4-labeling. The theorem holds.

Example 4.1: Following are labelings for some spiders of 3 even legs according to the proof above. The numbers listed in the parenthesis are the induced vertex colors.

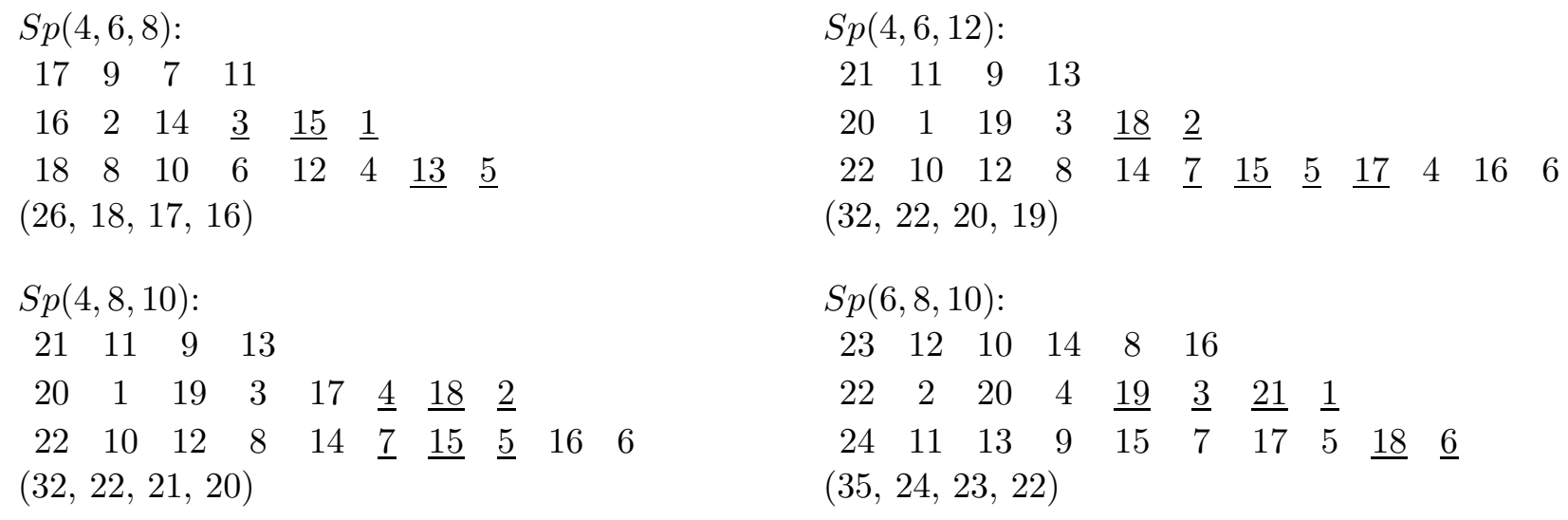




\section{$5 \quad$ Spiders with three odd legs}

Theorem 5.1. For $m, n \geq 1, k \geq 1, m+n+k$ even and $3 k \leq n+m, \chi_{l a}(\operatorname{Sp}(2 n+1,2 m+1, n+m+3 k+1))=4$.

Proof. Since $m+n \geq 3 k$, without loss of generality, we assume that $m>k$. Let $f$ be an edge labeling of $S p(2 n+1,2 m+1, n+m+3 k+1)$. Let $P_{2 n+2}=x_{1} \cdots x_{2 n+1} x_{2 n+2}, P_{2 m+2}=y_{1} \cdots y_{2 m+1} y_{2 m+2}$ and $P_{n+m+3 k+2}=z_{1} \cdots z_{n+m+3 k+1} z_{n+m+3 k+2}$. Let $u=x_{2 n+2}=y_{2 m+2}=z_{n+m+3 k+2}$. Now, $q=3 n+3 m+3 k+3$.

We label $P_{2 n+2}, P_{2 m+2}$ and $P_{n+m+3 k+2}$ as:

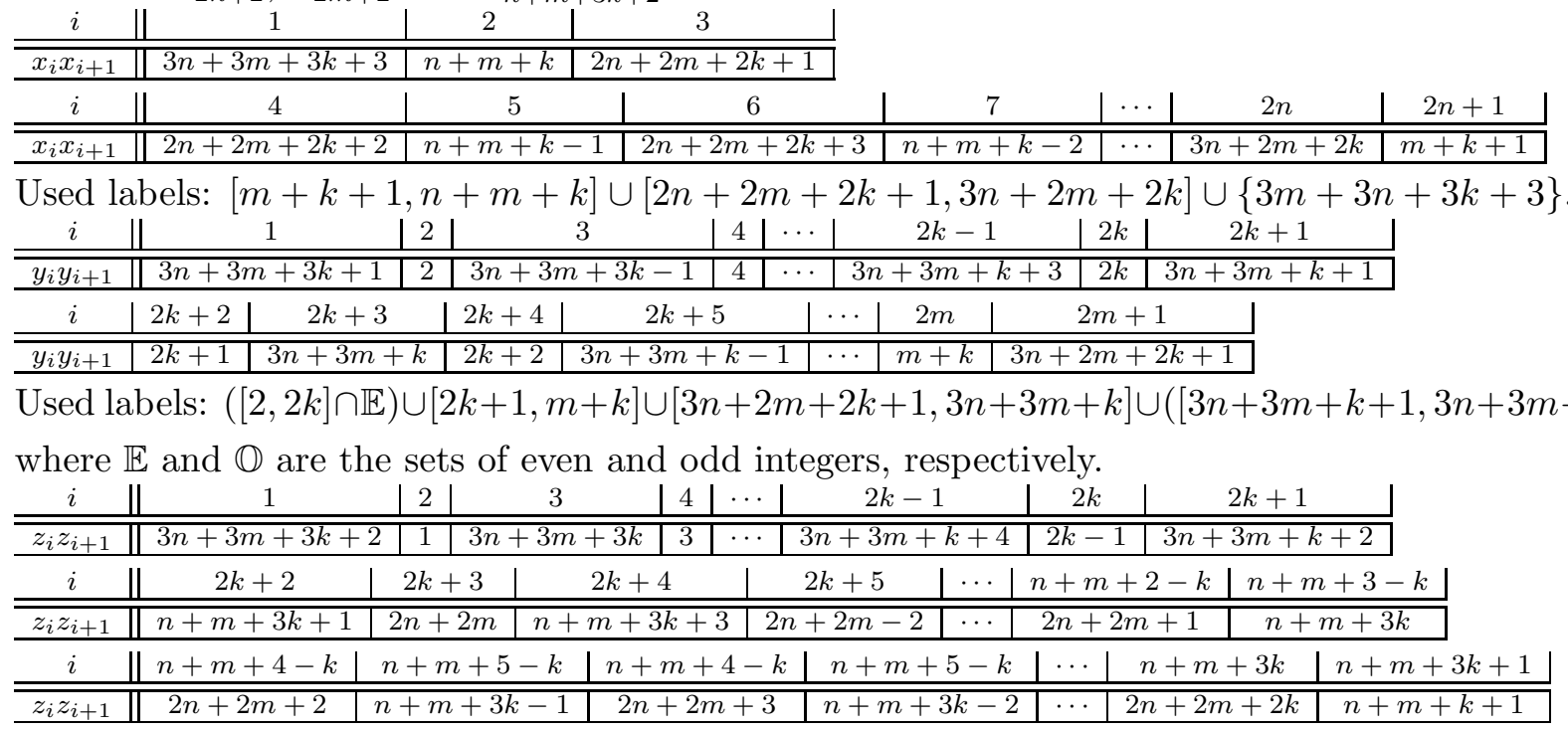

Used labels: $([1,2 k-1] \cap \mathbb{O}) \cup[n+m+k+1,2 n+2 m+2 k] \cup([3 n+3 m+k+2,3 n+3 m+3 k+2] \cap \mathbb{E})$.

Clearly $f$ is a bijection. Now $f^{+}\left(x_{2}\right)=f^{+}\left(x_{4}\right)=f^{+}\left(z_{2 k+2}\right)=f^{+}(u)=4 n+4 m+4 k+3$ and $f^{+}(w) \in\{3 n+3 m+3 k+3,3 n+3 m+3 k+2,3 n+3 m+3 k+1\}$ for other vertex $w$. Thus, $f$ is a required local antimagic 4-coloring.

Example 5.1: $S p(17,15,25)(n=8, m=7, k=3)$ :

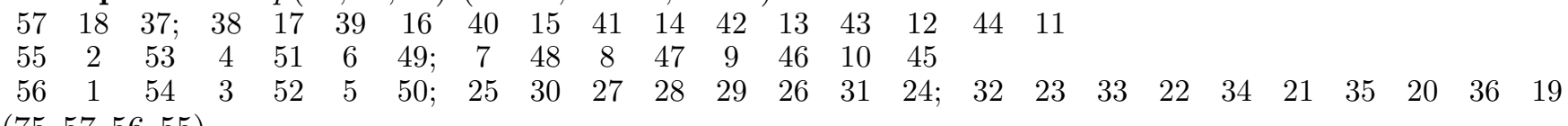

$(75,57,56,55)$

Theorem 5.2. For $m \geq 2, n \geq 1$ and $m+n \geq 4$ even, $\chi_{l a}(S p(2 n+1,2 m+1, n+m+1))=4$.

Proof. Let $f$ be a bijective edge labeling of $S p(2 n+1,2 m+1, n+m+1)$. Let $P_{2 n+2}=x_{1} \cdots x_{2 n+1} x_{2 n+2}$, $P_{2 m+2}=y_{1} \cdots y_{2 m+1} y_{2 m+2}$ and $P_{n+m+2}=z_{1} \cdots z_{n+m+1} z_{n+m+2}$. Let $u=x_{2 n+2}=y_{2 m+2}=z_{n+m+2}$. Now, $q=3 n+3 m+3$. We label $P_{2 n+2}, P_{2 m+2}$ and $P_{n+m+2}$ as:

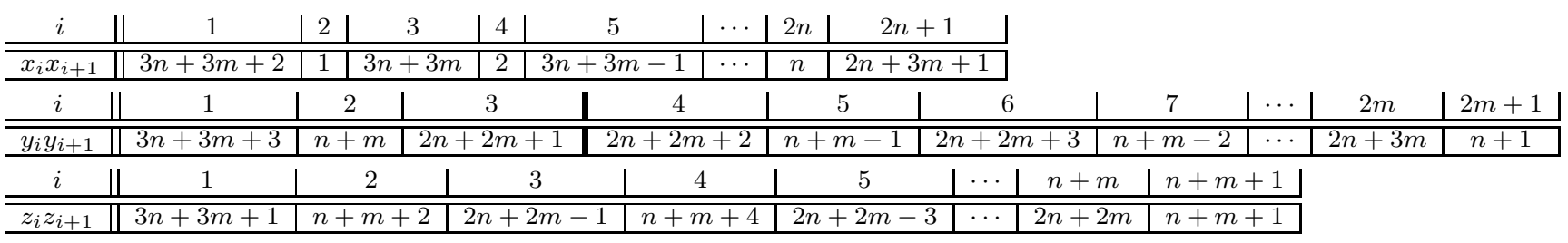

Now $f^{+}\left(y_{4}\right)=f^{+}(u)=4 n+4 m+3$ and $f^{+}(w) \in\{3 n+3 m+3,3 n+3 m+2,3 n+3 m+1\}$ for other vertex $w$. Thus, $f$ is a required local antimagic 4-coloring. 
Example 5.2: $\quad S p(9,17,13)$ :

$\begin{array}{ccccccccccccccccc}38 & 1 & 36 & 2 & 35 & 3 & 34 & 4 & 33 & & & & & & & \\ 39 & 12 & 25 & 26 & 11 & 27 & 10 & 28 & 9 & 29 & 8 & 30 & 7 & 31 & 6 & 32 & 5 \\ 37 & 14 & 23 & 16 & 21 & 18 & 19 & 20 & 17 & 22 & 15 & 24 & 13 & & & & \\ (37,38,39,51) & & & & & & & & & & & & & \end{array}$

By substituting $m=n+2$ or $m=k n$, where $k \geq 1$ and $(k+1) n$ is even, in Theorem 5.2 , we have

Corollary 5.3. For $n, k \geq 1$, $\chi_{l a}(S p(2 n+1,2 n+3,2 n+5))=\chi_{l a}(S p(2 n+1,2 k n+1,(k+1) n+1))=4$, where $(k+1) n$ is even.

Theorem 5.4. For $n \geq 0$ and $m \geq 1, \chi_{l a}(S p(2 n+1,2 m+1,2 m+1))=4$.

Proof. Let $f$ be a required labeling. Let $P_{2 n+2}=x_{1} \cdots x_{2 n+1} x_{2 n+2}, P_{2 m+2}=y_{1} \cdots y_{2 m+1} y_{2 m+2}$ and $P_{2 h+2}=$ $z_{1} \cdots z_{2 h+1} z_{2 h+2}$. Let $u=x_{2 n+2}=y_{2 m+2}=z_{2 h+2}$. We label $P_{2 n+2}$ and $P_{2 m+2}$ as:

\begin{tabular}{c||c|c|c|c|c|c|c|c|}
$i$ & 1 & 2 & 3 & 4 & $\cdots$ & $2 n-1$ & $2 n$ & $2 n+1$ \\
\hline \hline$x_{i} x_{i+1}$ & $q-1$ & 1 & $q-2$ & 2 & $\cdots$ & $q-n$ & $n$ & $q-n-1$ \\
\hline
\end{tabular}

When $n=0$. The above table has only one column.

\begin{tabular}{c||c|c|c|c|c|c|c|c|}
$i$ & 1 & 2 & 3 & 4 & $\cdots$ & $2 m-1$ & $2 m$ & $2 m+1$ \\
\hline \hline$y_{i} y_{i+1}$ & $n+1$ & $q-n-2$ & $n+2$ & $q-n-3$ & $\cdots$ & $n+m$ & $q-n-m-1$ & $n+m+1$ \\
\hline
\end{tabular}

\begin{tabular}{c||c||c|c|c|c|c|c|}
$i$ & 1 & 2 & 3 & $\cdots$ & $2 h-1$ & $2 h$ & $2 h+1$ \\
\hline \hline$z_{i} z_{i+1}$ & $q$ & $q-n-m-2$ & $n+m+2$ & $\cdots$ & $n+m+h$ & $q-n-m-h-1$ & $n+m+h+1$ \\
\hline
\end{tabular}

Now $f^{+}(u)=q+n+2 m+h+1=3 n+4 m+3 h+4, f^{+}\left(z_{2}\right)=2 q-n-m-2=3 n+3 m+4 h+4$, $f^{+}\left(y_{1}\right)=n+1$ and $f^{+}(w) \in\{q, q-1\}$ for other vertex $w$. Thus, when $m=h$ we have the result.

\section{Example 5.3:}

$S p(5,5,9) \cong S p(9,5,5):$

$\begin{array}{cccccccccccccccccc}18 & 1 & 17 & 2 & 16 & 3 & 15 & 4 & 14 & 24 & 1 & 23 & 2 & 22 & 3 & 21 & & \\ 5 & 13 & 6 & 12 & 7 & & & & & 4 & 20 & 5 & 19 & 6 & 18 & 7 & 17 & 8 \\ 19 & 11 & 8 & 10 & 9 & & & 25 & 16 & 9 & 15 & 10 & 14 & 11 & 13 & 12 \\ (30, & 19, & 18,5) & & & & & & \end{array}$

Starting from the labeling of $S p(2 n+1,2 m+1,2 m+1)$ defined in the proof of Theorem 5.4 for $m>n$, we move the $2 n+2$ right most numbers of the second leg to the end of first leg. We then can get a required labeling for $S p(4 n+3,2 m-2 n-1,2 m+1)$.

Namely, change the following assignment

\begin{tabular}{c||c|c|c|c|c|c|c|}
$i$ & 1 & 2 & 3 & $\cdots$ & $2 n-1$ & $2 n$ & $2 n+1$ \\
\hline \hline$x_{i} x_{i+1}$ & $q-1$ & 1 & $q-2$ & $\cdots$ & $q-n$ & $n$ & $q-n-1$ \\
\hline
\end{tabular}

\begin{tabular}{c||c|c|c|c||c|c|c|c|c|}
$i$ & 1 & 2 & $\cdots$ & $2(m-n)-1$ & $2(m-n)$ & $\cdots$ & $2 m-1$ & $2 m$ & $2 m+1$ \\
\hline \hline$y_{i} y_{i+1}$ & $n+1$ & $q-n-2$ & $\cdots$ & $m$ & $q-m-1$ & $\cdots$ & $n+m$ & $q-n-m-1$ & $n+m+1$ \\
\hline
\end{tabular}

to

\begin{tabular}{c||c|c|c|c|c|c|c||c|c|c|c|c|}
$j$ & 1 & 2 & 3 & $\cdots$ & $2 n-1$ & $2 n$ & $2 n+1$ & $2 n+2$ & $\cdots$ & $4 n+1$ & $4 n+2$ & $4 n+3$ \\
\hline \hline$v_{j} v_{j+1}$ & $q-1$ & 1 & $q-2$ & $\cdots$ & $q-n$ & $n$ & $q-n-1$ & $q-m-1$ & $\cdots$ & $n+m$ & $q-n-m-1$ & $n+m+1$ \\
\hline
\end{tabular}




\begin{tabular}{c||c|c|c|c|}
$i$ & 1 & 2 & $\cdots$ & $2(m-n)-1$ \\
\hline \hline$w_{i} w_{i+1}$ & $n+1$ & $q-n-2$ & $\cdots$ & $m$ \\
\hline
\end{tabular}

and keep the labeling of the leg $P_{2 m+2}=z_{1} \cdots z_{2 m+2}$ as

\begin{tabular}{c||c||c|c|c|c|c|c|}
$j$ & 1 & 2 & 3 & $\cdots$ & $2 m-1$ & $2 m$ & $2 m+1$ \\
\hline \hline$z_{j} z_{j+1}$ & $q$ & $q-n-m-2$ & $n+m+2$ & $\cdots$ & $n+2 m$ & $q-n-2 m-1$ & $n+2 m+1$ \\
\hline
\end{tabular}

Hence we have a labeling, say $g$, for $P_{4 n+4}=v_{1} \cdots v_{4 n+4}, P_{2 m-2 n}=w_{1} \cdots w_{2 m-2 n}$ and $P_{2 m+2}=z_{1} \cdots z_{2 m+2}$. Now $g^{+}(u)=2 n+4 m+2=q-1, g^{+}\left(z_{2}\right)=2 q-n-m-2=3 n+7 m+4=g^{+}\left(v_{2 n+2}\right), g^{+}\left(y_{1}\right)=n+1$ and $g^{+}(v) \in\{q, q-1\}$ for other vertex $v$. So we have a local antimagic 4-labeling for $S p(2(m-n)-1,2 m+$ $1,4 n+3)$. After rewriting the parameters we have

Theorem 5.5. For $m>n \geq 0, \chi_{l a}(S p(2 n+1,2 m+1,4(m-n)-1))=4$.

\section{Example 5.4:}

Starting from the labeling of $S p(3,9,9)$ : $\begin{array}{lll}20 & 1 & 19\end{array}$

$\begin{array}{ccccccccc}2 & 18 & 3 & 17 & 4 & \frac{16}{12} & \frac{5}{9} & \frac{15}{11} & \underline{6} \\ 21 & 14 & 7 & 13 & 8 & \end{array}$

we get $S p(7,5,9) \cong S p(5,7,9)$ :

$\begin{array}{ccccccccc}20 & 1 & 19 & \frac{16}{17} & \frac{5}{4} & \underline{15} & \underline{6} & & \\ 2 & 18 & 3 & 17 & & & & \\ 21 & 14 & 7 & 13 & 8 & 12 & 9 & 11 & 10\end{array}$

$(2,10,21,35)$

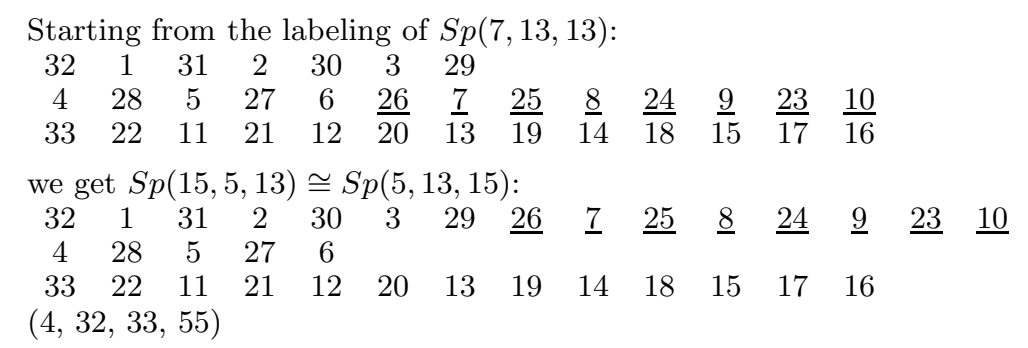

Theorem 5.6. For $m \geq 4, \chi_{l a}(S p(2 m+1,2 m+3,4 m-3))=4$.

Proof. Let $f$ be a bijective edge labeling of $S p(2 m+1,2 m+3,4 m-3)$. Let $P_{2 m+2}=x_{1} \cdots x_{2 m+1} x_{2 m+2}$, $P_{2 m+4}=y_{1} \cdots y_{2 m+3} y_{2 m+4}$ and $P_{4 m-2}=z_{1} \cdots z_{4 m-3} z_{4 m-2}$. Let $u=x_{2 m+2}=y_{2 m+4}=z_{4 m-2}$. Now, $q=8 m+1$. We label $P_{2 m+2}, P_{2 m+4}$ and $P_{4 m-2}$ as:

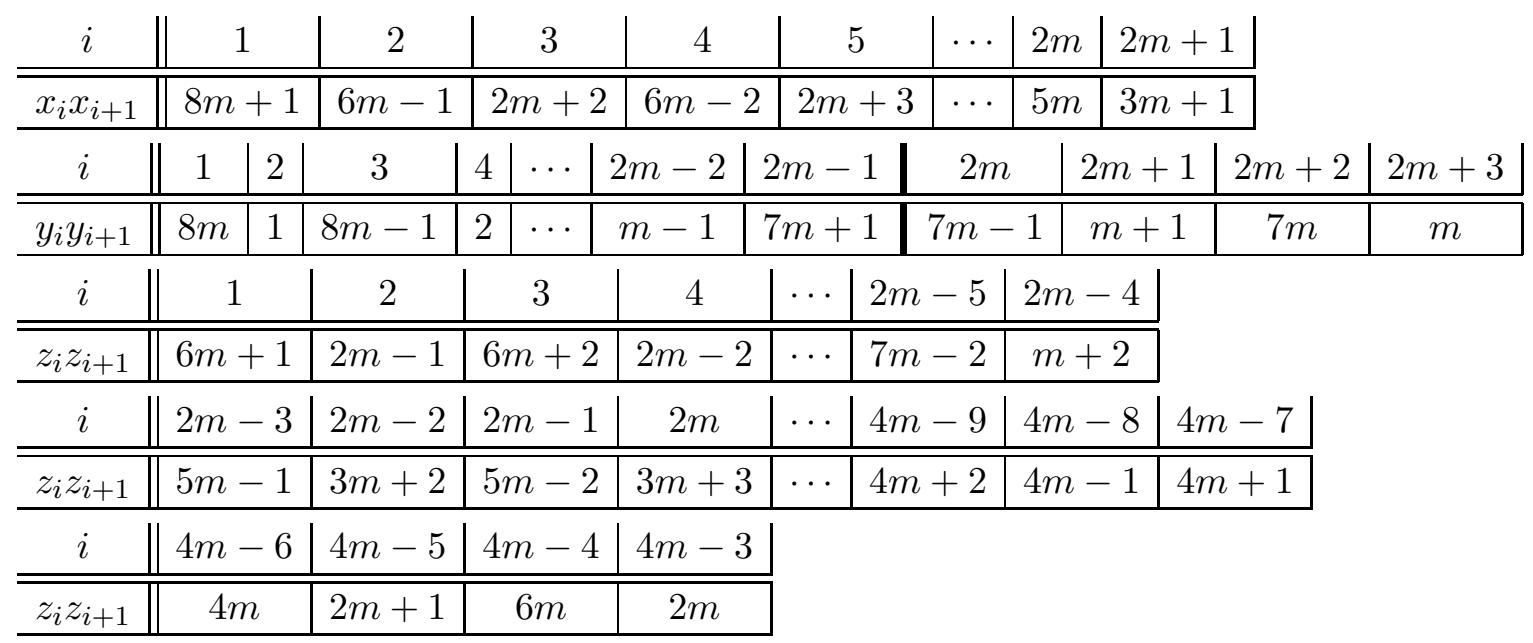

Now $f^{+}\left(x_{2}\right)=f^{+}(u)=14 m$ and $f^{+}(w) \in\{8 m+1,8 m, 6 m+1\}$ for other vertex $w$. Thus, $f$ is a required local antimagic 4-coloring.

Example 5.5: $\quad S p(11,13,17)$ :

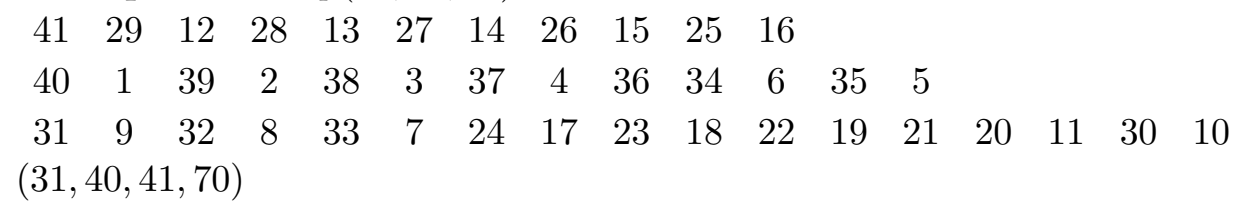


Observe that if we move the last four labels of $P_{2 m+2}$ (in the proof of Theorem 5.6) to the right end of $P_{2 m+4}$ to form a labeling of $P_{2 m+8}$, we then obtain a local antimagic 4-labeling for $S p(2 m-3,2 m+7,4 m-3)$. Renaming the parameters, we get the following theorem.

Theorem 5.7. For $m \geq 2, \chi_{l a}(S p(2 m+1,2 m+11,4 m+5))=4$.

Proof. Let $f$ be a bijective edge labeling of $S p(2 m+1,2 m+11,4 m+5)$. Now, $q=8 m+17$. Let $u=x_{2 m+2}=$ $y_{2 m+12}=z_{4 m+6}$. The labelings of $P_{2 m+2}=x_{1} \cdots x_{2 m+2}, P_{2 m+12}=y_{1} \cdots y_{2 m+12}$ and $P_{4 m+6}=z_{1} \cdots z_{4 m+6}$ are given as follows.

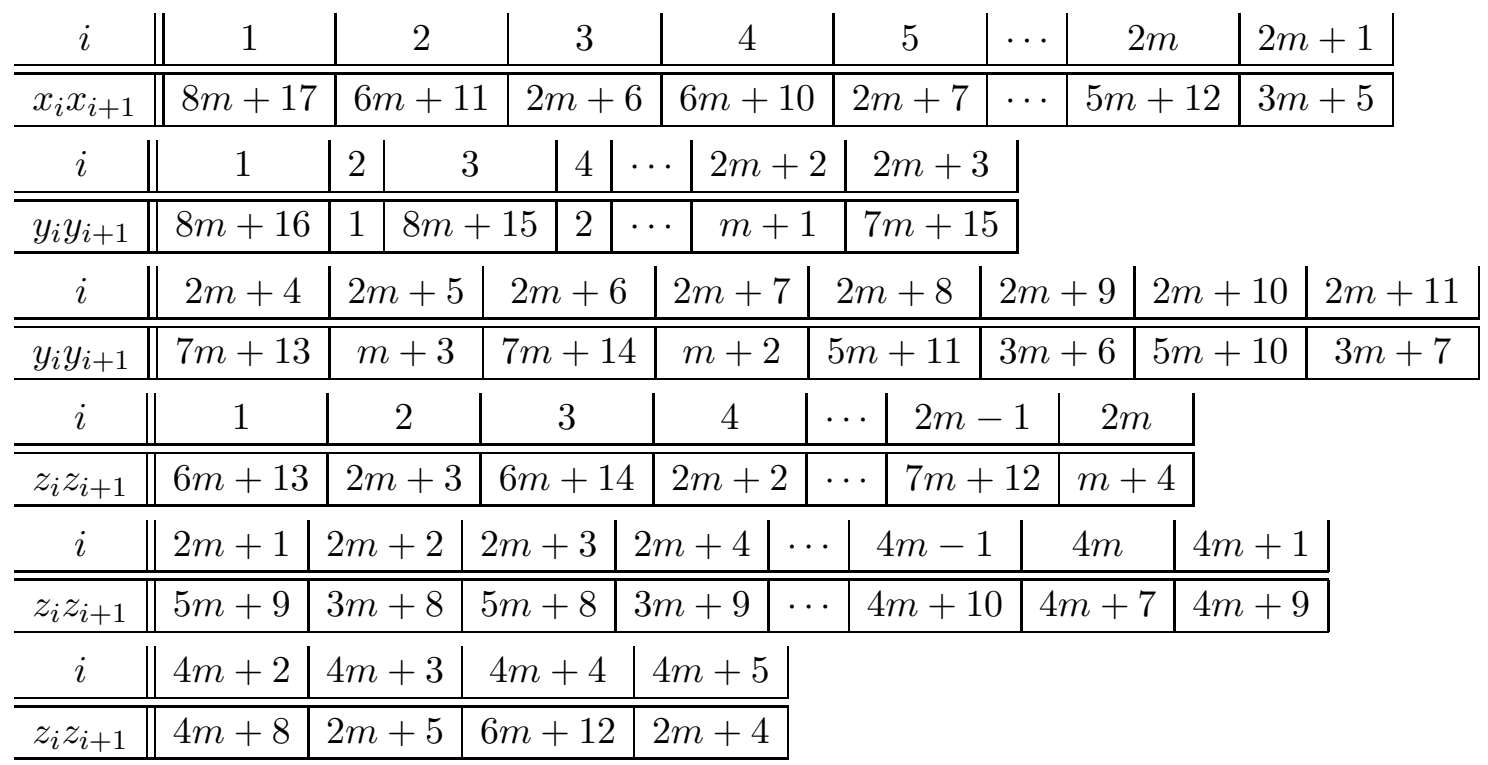

Now, $f^{+}\left(x_{2}\right)=f^{+}\left(y_{2 m+4}\right)=14 m+28$ and $f^{+}(w) \in\{8 m+17,8 m+16,6 m+1\}$ for other vertex $w$. Thus, $f$ is a required local antimagic 4 -coloring.

Example 5.6: $\quad S p(9,19,21)$ :

$$
\begin{aligned}
& \begin{array}{lllllllll}
49 & 35 & 14 & 34 & 15 & 33 & 16 & 32 & 17
\end{array} \\
& \begin{array}{lllllllllllllllllll}
48 & 1 & 47 & 2 & 46 & 3 & 45 & 4 & 44 & 5 & 43 & 41 & 7 & 42 & 6 & 31 & 18 & 30 & 19
\end{array} \\
& \begin{array}{lllllllllllllllllllll}
37 & 11 & 38 & 10 & 39 & 9 & 40 & 8 & 29 & 20 & 28 & 21 & 27 & 22 & 26 & 23 & 25 & 24 & 13 & 36 & 12
\end{array} \\
& (37,48,49,84)
\end{aligned}
$$

Theorem 5.8. For $h \geq 0, m \geq 1, \chi_{l a}(S p(3,2 m+1,2 m+2 h+1))=4$.

Proof. Let $f$ be a required labeling. We first consider $h \geq 2$. Let $P_{2 m+2}^{(1)}=x_{1} \cdots x_{2 m+1} x_{2 m+2}, P_{2 m+2}^{(2)}=$ $y_{1} \cdots y_{2 m+1} y_{2 m+2}$ and $P_{4}=w_{1} w_{2} w_{3} w_{4}$. Firstly, let $f\left(w_{1} w_{2}\right)=q$.

We label $P_{2 m+2}^{(j)}$ by integers in $[1,2 m] \cup[q-2 m-2, q-1]$ :

\begin{tabular}{c||c|c|c|c|c|c|c|c|}
$i$ & 1 & 2 & 3 & 4 & $\cdots$ & $2 m-1$ & $2 m$ & $2 m+1$ \\
\hline \hline$x_{i} x_{i+1}$ & $q-1$ & 1 & $q-3$ & 3 & $\cdots$ & $q-2 m+1$ & $2 m-1$ & $q-2 m-1$ \\
\hline$y_{i} y_{i+1}$ & $q-2$ & 2 & $q-4$ & 4 & $\cdots$ & $q-2 m$ & $2 m$ & $q-2 m-2$ \\
\hline
\end{tabular}

Suppose $h=2 k$ and $k \geq 1$. Now, we have $S p(3,2 m+1,2 m+4 k+1)$ and $q=4 m+4 k+5$. Let $Q_{2 k+1}=z_{1} \cdots z_{2 k+1}$ and $R_{2 k+1}=v_{1} \cdots v_{2 k+1}$. We label $Q_{2 k+1}$ by in integers in $[2 m+1,2 m+k] \cup[2 m+$ $3 k+3,2 m+4 k+2=q-2 m-3]:$

\begin{tabular}{c||c|c|c|c|c|c|c|c|c|}
$i$ & 1 & 2 & 3 & 4 & $\cdots$ & $2 k-3$ & $2 k-2$ & $2 k-1$ & $2 k$ \\
\hline \hline$z_{i} z_{i+1}$ & $2 m+1$ & $q-2 m-3$ & $2 m+2$ & $q-2 m-4$ & $\cdots$ & $2 m+k-1$ & $2 m+3 k+4$ & $2 m+k$ & $2 m+3 k+3$ \\
\hline
\end{tabular}


We label $R_{2 k+1}$ by integers in $[2 m+k+2,2 m+3 k+1]$ :

\begin{tabular}{c||c|c|c|c|c|c|c|c|c|}
$i$ & 1 & 2 & 3 & 4 & $\cdots$ & $2 k-3$ & $2 k-2$ & $2 k-1$ & $2 k$ \\
\hline \hline$v_{i} v_{i+1}$ & $2 m+k+2$ & $2 m+3 k+1$ & $2 m+k+3$ & $2 m+3 k$ & $\cdots$ & $2 m+k-1$ & $2 m+2 k+3$ & $2 m+2 k+1$ & $2 m+2 k+2$ \\
\hline
\end{tabular}

Let $P_{2 m+2 h+2}=P_{2 m+2}^{(2)} Q_{2 k+1} R_{2 k+1}$. Lastly, let $f\left(w_{2} w_{3}\right)=2 m+3 k+2$ and $f\left(w_{3} w_{4}\right)=2 m+k+1$. It is easy to check that $f^{+}(u)=q+2 m+3 k+2=f^{+}\left(w_{2}\right)$ and each other vertex has induced label in $\{q-2, q-1, q\}$. Thus, $f$ is a local antimagic 4-labeling.

Suppose $h=2 k+1$ and $k \geq 1$. Now, we have $S p(3,2 m+1,2 m+4 k+3)$ and $q=4 m+4 k+7$. Let $Q_{2 k+3}=z_{1} \cdots z_{2 k+3}$ and $R_{2 k+1}=v_{1} \cdots v_{2 k+1}$. We label $Q_{2 k+3}$ by in integers in $[2 m+1,2 m+k+1] \cup[2 m+$ $3 k+4,2 m+4 k+4=q-2 m-3]:$

\begin{tabular}{c||c|c|c|c|c|c|c|c|c|}
$i$ & 1 & 2 & 3 & 4 & $\cdots$ & $2 k-1$ & $2 k$ & $2 k+1$ & $2 k+2$ \\
\hline \hline$z_{i} z_{i+1}$ & $2 m+1$ & $q-2 m-3$ & $2 m+2$ & $q-2 m-4$ & $\cdots$ & $2 m+k$ & $2 m+3 k+5$ & $2 m+k+1$ & $2 m+3 k+4$ \\
\hline
\end{tabular}

We label $R_{2 k+1}$ by integers in $[2 m+k+3,2 m+3 k+2]$ :

\begin{tabular}{c||c|c|c|c|c|c|c|c|c}
$i$ & 1 & 2 & 3 & 4 & $\cdots$ & $2 k-3$ & $2 k-2$ & $2 k-1$ & $2 k$ \\
\hline \hline$v_{i} v_{i+1}$ & $2 m+k+3$ & $2 m+3 k+2$ & $2 m+k+4$ & $2 m+3 k+1$ & $\cdots$ & $2 m+k+1$ & $2 m+2 k+4$ & $2 m+2 k+2$ & $2 m+2 k+3$ \\
\hline
\end{tabular}

Let $P_{2 m+2 h+2}=P_{2 m+2}^{(1)} Q_{2 k+3} R_{2 k+1}$. Lastly, let $f\left(w_{2} w_{3}\right)=2 m+3 k+3$ and $f\left(w_{3} w_{4}\right)=2 m+k+2$. It is easy to check that $f^{+}(u)=q+2 m+3 k+3=f^{+}\left(w_{2}\right)$ and each other vertex has induced label in $\{q-2, q-1, q\}$. Thus, $f$ is a local antimagic 4-labeling.

For $h=1$, let $P_{2 m+4}=x_{1} \cdots x_{2 m+4}, P_{2 m+2}=y_{1} \cdots y_{2 m+2}$ and $P_{4}=w_{1} w_{2} w_{3} w_{4}$. Now $q=4 m+7$. We label $P_{2 m+4}$ and $P_{2 m+2}$ as:

\begin{tabular}{c||c|c|c|c|c|c|c|c|c|c|c|c|c|}
$i$ & 1 & 2 & 3 & 4 & 5 & 6 & 7 & $\cdots$ & $2 m-1$ & $2 m$ & $2 m+1$ & $2 m+2$ & $2 m+3$ \\
\hline \hline$x_{i} x_{i+1}$ & $4 m+7$ & $4 m+6$ & 1 & $2 m+1$ & $2 m+6$ & $2 m-1$ & $2 m+8$ & $\cdots$ & $\cdots$ & 5 & $4 m+2$ & 3 & $4 m+4$ \\
\hline$y_{i} y_{i+1}$ & $4 m+5$ & 2 & $4 m+3$ & 4 & $4 m+1$ & $\cdots$ & $\cdots$ & $\cdots$ & $2 m+7$ & $2 m$ & $2 m+5$ & & \\
\hline
\end{tabular}

Let $f\left(w_{1} w_{2}\right)=2 m+2, f\left(w_{2} w_{3}\right)=2 m+3$ and $f\left(w_{3} w_{4}\right)=2 m+4$. Then the induced vertex labels are $8 m+13,4 m+7,4 m+5$ and $2 m+2$. Thus, $f$ is a local antimagic 4-labeling.

For $h=0$, let $P_{2 m+2}=x_{1} \cdots x_{2 m+2}, Q_{2 m+2}=y_{1} \cdots y_{2 m+2}$ and $P_{4}=w_{1} w_{2} w_{3} w_{4}$.

We label $P_{2 m+2}$ and $Q_{2 m+2}$ by

\begin{tabular}{c||c|c|c|c|c|c|c|c|c|c|}
$i$ & 1 & 2 & 3 & 4 & 5 & $\cdots$ & $2 m-2$ & $2 m-1$ & $2 m$ & $2 m+1$ \\
\hline \hline$x_{i} x_{i+1}$ & $4 m+2$ & 2 & $4 m$ & 4 & $4 m-2$ & $\cdots$ & $2 m-2$ & $2 m+4$ & $2 m$ & $2 m+2$ \\
\hline$y_{i} y_{i+1}$ & $4 m+5$ & $4 m+1$ & 3 & $4 m-1$ & 5 & $\cdots$ & $2 m+5$ & $2 m-1$ & $2 m+3$ & $2 m+1$ \\
\hline
\end{tabular}

Let $f\left(w_{1} w_{2}\right)=4 m+4, f\left(w_{2} w_{3}\right)=1$ and $f\left(w_{3} w_{4}\right)=4 m+3$. Thus, $f$ is a local antimagic 4-labeling.

Hence the proof is completed.

Theorem 5.9. For $h \geq 3, \chi_{l a}(S p(5,2 m+1,2 h+1))=4$.

Proof. Let $P_{6}=x_{1} \ldots x_{6}, P_{2 m+2}=y_{1} \cdots y_{2 m+2}$ and $P_{2 h+2}=z_{1} z_{2} \cdots z_{2 h+2}$ with $u=x_{6}=y_{12}=z_{2 h+2}$. Now, $q=2 m+2 h+7$. Let $f$ be a required labeling.

The leg $P_{6}$ is labeled as follows.

\begin{tabular}{c||c|c|c|c|c|}
$i$ & 1 & 2 & 3 & 4 & 5 \\
\hline \hline$x_{i} x_{i+1}$ & $q-1$ & 1 & $q-2$ & $\frac{q+1}{2}$ & $\frac{q-1}{2}$ \\
\hline
\end{tabular}

The leg $P_{2 m+2}$ is labeled as follows.

\begin{tabular}{c||c|c|c|c|c|c|c|c|}
$j$ & 1 & 2 & 3 & 4 & 5 & $\cdots$ & $2 m$ & $2 m+1$ \\
\hline \hline$y_{j} y_{j+1}$ & $q$ & $\frac{q-3}{2}$ & $\frac{q+3}{2}$ & $\frac{q-5}{2}$ & $\frac{q+5}{2}$ & $\cdots$ & $\frac{q-1}{2}-m$ & $\frac{q+1}{2}+m$ \\
\hline
\end{tabular}


The leg $P_{2 h+2}$ is labeled as follows.

\begin{tabular}{c||c|c|c|c|c|c|c|}
$k$ & 1 & 2 & 3 & 4 & $\cdots$ & $2 h$ & $2 h+1$ \\
\hline \hline$z_{k} z_{k+1}$ & 2 & $q-3$ & 3 & $q-4$ & $\cdots$ & $q-h-2$ & $h+2$ \\
\hline
\end{tabular}

Note that $h+2=\frac{q-1}{2}-m-1$ and $q-h-2=\frac{q+1}{2}+m+1$. So $f$ is a bijection. Now, $f^{+}(u)=f^{+}\left(x_{4}\right)=$ $f^{+}\left(y_{2}\right)=\frac{3 q-3}{2}, f^{+}\left(z_{1}\right)=2$, and $f^{+}(w) \in\{q, q-1\}$ for other vertex $w$. The theorem holds.

Example 5.7: $S p(5,9,11)$ :

$\begin{array}{ccccccccccc}24 & 1 & 23 & 13 & 12 & & & & & & \\ 25 & 11 & 14 & 10 & 15 & 9 & 16 & 8 & 17 & & \\ 2 & 22 & 3 & 21 & 4 & 20 & 5 & 19 & 6 & 18 & 7\end{array}$

$(36,25,24,2)$

Theorem 5.10. For $m, h \geq 1, \chi_{l a}(S p(7,2 m+1,2 h+1))=4$.

Proof. Let $P_{8}=x_{1} \cdots x_{8}, P_{2 m+2}=y_{1} \cdots y_{2 m+2}, P_{2 h+1}=z_{1} \cdots z_{2 h+2}$, and $u=x_{8}=y_{2 m+2}=z_{2 h+2}$. Now, $q=2 m+2 h+9$. Let $f$ be a required labeling.

The leg $P_{8}$ is labeled as follows:

\begin{tabular}{c||c|c|c|c|c|c|c|}
$i$ & 1 & 2 & 3 & 4 & 5 & 6 & 7 \\
\hline \hline$x_{i} x_{i+1}$ & $q-2$ & 2 & $q-4$ & $q-1$ & 1 & 3 & $q-3$ \\
\hline
\end{tabular}

The leg $P_{2 m+2}$ is labeled by using all even numbers in $[4,2 m+3]$ and all odd numbers in $[q-2 m-4, q-6]=$ $[2 h+5]$ as follows:

\begin{tabular}{c||c|c|c|c|c|c|c|c|}
$j$ & 1 & 2 & 3 & 4 & 5 & $\cdots$ & $2 m$ & $2 m+1$ \\
\hline \hline$y_{i} y_{i+1}$ & 4 & $q-6$ & 6 & $q-8$ & 8 & $\cdots$ & $q-2 m-4$ & $2 m+4$ \\
\hline
\end{tabular}

The leg $P_{2 h+2}$ is labeled by using all odd numbers in $[5,2 h+3] \cup\{q\}$ and all even numbers in $[q-2 h-3, q-5]=$ $[2 m+6, q-5]$ as follows:

\begin{tabular}{c||c|c|c|c|c|c|c|c|}
$i$ & 1 & 2 & 3 & 4 & 5 & $\cdots$ & $2 h$ & $2 h+1$ \\
\hline \hline$z_{i} z_{i+1}$ & $q$ & $q-5$ & 5 & $q-7$ & 7 & $\cdots$ & $q-2 h-3$ & $2 h+3$ \\
\hline
\end{tabular}

Clearly, $f$ is a bijection. Now $f^{+}(u)=f^{+}\left(x_{4}\right)=f^{+}\left(z_{2}\right)=2 q-5, f\left(x_{6}\right)=4=f^{+}\left(y_{1}\right)$, and $f^{+}(w) \in\{q, q-2\}$ for other vertex $w$. The theorem holds.

Theorem 5.11. For $m \geq 2$, $\chi_{l a}(\operatorname{Sp}(9,11,2 m+1))=4$.

Proof. Let $f$ be an edge labeling of $S p(9,11,2 m+1)$. Let $P_{10}=x_{1} \cdots x_{10}, P_{12}=y_{1} \cdots y_{12}$ and $P_{2 m+2}=$ $z_{1} \cdots z_{2 m+2}$. Let $x_{10}=y_{12}=z_{2 m+2}$. Now, $q=2 m+21$. We label $P_{10}, P_{12}$ and $P_{2 m+2}$ as:

\begin{tabular}{c||c|c|c|c|c|c|c|c|c|c|c|c|c}
$i$ & 1 & 2 & 3 & 4 & 5 & 6 & 7 & 8 & 9 & \multirow{2}{*}{} \\
\hline \hline$x_{i} x_{i+1}$ & $2 m+21$ & $2 m+14$ & 6 & $2 m+15$ & 5 & $m+9$ & $m+12$ & $m+8$ & $m+13$ & \\
\hline$i$ & 1 & 2 & 3 & 4 & 5 & 4 & 6 & 7 & 8 & 9 & 10 & 11 \\
\hline \hline$y_{i} y_{i+1}$ & $2 m+20$ & 1 & $2 m+19$ & 2 & $2 m+18$ & $2 m+17$ & 3 & $m+11$ & $m+10$ & 4 & $2 m+16$ \\
\hline$i$ & 1 & 2 & 3 & 4 & 5 & 6 & 7 & 8 & 9 & 10 & 11 \\
\hline \hline$z_{i} z_{i+1}$ & $m+14$ & $m+7$ & 7 & $2 m+13$ & 8 & $2 m+12$ & $\cdots$ & $m+16$ & $m+5$ & $m+15$ & $m+6$ \\
\hline
\end{tabular}

Clearly $f$ is a bijection. Now, $f^{+}\left(x_{2}\right)=f^{+}\left(y_{6}\right)=f^{+}(u)=4 m+35$ and $f^{+}(w) \in\{m+14,2 m+20,2 m+$ $21\}$ for other vertex $w$. Thus, $f$ is a required local antimagic 4-coloring. 
Theorem 5.12. For $m, n \geq 2, \chi_{l a}(S p(13,2 m+1,2 n+1))=4$.

Proof. Let $f$ be an edge labeling of $S p(13,2 m+1,2 n+1)$. Let $P_{14}=x_{1} \cdots x_{14}, P_{2 m+2}=y_{1} \cdots y_{2 m+2}$ and $P_{2 n+2}=z_{1} \cdots z_{2 n+2}$. Let $x_{14}=y_{2 m+2}=z_{2 n+2}$. Now, $q=2 m+2 n+15$. We label $P_{14}, P_{2 m+2}$ and $P_{22+2}$ as: \begin{tabular}{c||c|c|c|c|c|c|c|}
$i$ & 1 & 2 & 3 & 4 & 5 & 7 \\
\hline \hline$x_{i} x_{i+1}$ & $2 m+2 n+13$ & 2 & $2 m+2 n+11$ & 4 & 3 & $2 m+2 n+12$ & $2 m+2 n+10$ \\
\hline$x_{i+7} x_{i+8}$ & 5 & $2 m+2 n+8$ & $2 m+2 n+14$ & 1 & 6 & $2 m+2 n+9$ & \\
\hline
\end{tabular}

\begin{tabular}{c||c|c|c|c|c|c|c|c|}
\hline$i$ & 1 & 2 & 3 & 4 & 5 & 6 & 7 & 8 \\
\hline \hline$y_{i} y_{i+1}$ & $2 m+2 n+15$ & $2 m+2 n+7$ & 8 & $2 m+2 n+5$ & 10 & $\cdots$ & $2 n+9$ & $2 m+6$ \\
\hline
\end{tabular}

\begin{tabular}{c||c|c|c|c|c|c|c|c|}
\hline$i$ & 1 & 2 & 3 & 4 & 5 & 6 & 7 & 8 \\
\hline \hline$z_{i} z_{i+1}$ & 7 & $2 m+2 n+6$ & 9 & $2 m+2 n+4$ & $\cdots$ & $2 n+5$ & $2 m+8$ & $2 n+7$ \\
\hline
\end{tabular}

Clearly $f$ is a bijection. Now, $f^{+}\left(x_{7}\right)=f^{+}(u)=4 m+4 n+22$ and $f^{+}(w) \in\{7,2 m+2 n+13,2 m+2 n+15\}$ for other vertex $w$. Thus, $f$ is a required local antimagic 4-coloring.

Example 5.8: $S p(13,9,15)$

$\begin{array}{ccccccccccccccc}35 & 2 & 33 & 4 & 3 & 34 & 32 & 5 & 30 & 36 & 1 & 6 & 31 & & \\ 37 & 29 & 8 & 27 & 10 & 25 & 12 & 23 & 14 & & & & & & \\ 7 & 28 & 9 & 26 & 11 & 24 & 13 & 22 & 15 & 20 & 17 & 18 & 19 & 16 & 21 \\ (7,35,37,66) & & & & & & & & & & & & \end{array}$

Conjecture 5.1. For $d \geq 3, y_{1}, y_{2}, \ldots, y_{d} \geq 2$ and $d(d+1) \leq 2(2 q-1), \chi_{l a}\left(S p\left(y_{1}, y_{2}, \ldots, y_{d}\right)\right)=d+1$ except $S p\left(2^{[n]}, 3^{[m]}\right)$ for $(n, m) \in\{(4,0),(5,0),(6,0),(0,10),(1,8),(1,9),(2,7),(2,8),(3,5),(3,6),(4,4),(4,5),(5,3)\}$.

\section{Appendix}

Following are local antimagic $(n+m+1)$-labelings of the spider $S p\left(2^{[n]}, 3^{[m]}\right)$, for some $(n, m)$. The numbers listed in the parenthesis are the induced vertex colors.
$(n, m)=(0,3)$ :
$(n, m)=(0,4)$ :
$8 \begin{array}{lll}8 & 1 & 7\end{array}$
$\begin{array}{lll}12 & 5 & 4\end{array}$
$\begin{array}{lll}6 & 2 & 4\end{array}$
$\begin{array}{lll}11 & 6 & 3\end{array}$
$\begin{array}{lll}9 & 5 & 3\end{array}$
$\begin{array}{lll}10 & 7 & 2\end{array}$
$\begin{array}{lll}9 & 8 & 1\end{array}$

$(14,9,8,6)$

$(n, m)=(0,5):$

$(17,12,11,10,9)$

$$
\begin{array}{ccc}
15 & 6 & 5 \\
14 & 7 & 4 \\
13 & 8 & 3 \\
12 & 9 & 2 \\
11 & 10 & 1
\end{array}
$$

$(21,15,14,13,12,11)$

$(n, m)=(0,7)$ :
$\begin{array}{lll}21 & 9 & 8\end{array}$
$\begin{array}{lll}20 & 10 & 7\end{array}$
$\begin{array}{lll}19 & 11 & 5\end{array}$
$\begin{array}{lll}18 & 12 & 4\end{array}$
$\begin{array}{lll}17 & 13 & 3\end{array}$
$\begin{array}{lll}16 & 14 & 2\end{array}$
$\begin{array}{lll}6 & 15 & 1\end{array}$

$(30,21,20,19,18,17,16,6)$ $(n, m)=(0,6)$ :

$\begin{array}{lll}18 & 8 & 7\end{array}$

$\begin{array}{lll}17 & 9 & 6\end{array}$

$\begin{array}{lll}16 & 10 & 5\end{array}$

$\begin{array}{lll}15 & 11 & 4\end{array}$

$\begin{array}{lll}14 & 12 & 3\end{array}$

$\begin{array}{lll}2 & 13 & 1\end{array}$

$(26,18,17,16,15,14,2)$

$(n, m)=(0,8)$ :

$\begin{array}{lll}24 & 13 & 6\end{array}$

$\begin{array}{lll}23 & 14 & 5\end{array}$

$\begin{array}{lll}22 & 15 & 4\end{array}$

$\begin{array}{lll}21 & 16 & 3\end{array}$

$\begin{array}{lll}20 & 17 & 2\end{array}$

$\begin{array}{lll}19 & 18 & 1\end{array}$

$\begin{array}{lll}10 & 12 & 7\end{array}$

$\begin{array}{lll}8 & 11 & 9\end{array}$

$(37,24,23,22,21,20,19,10,8)$ 


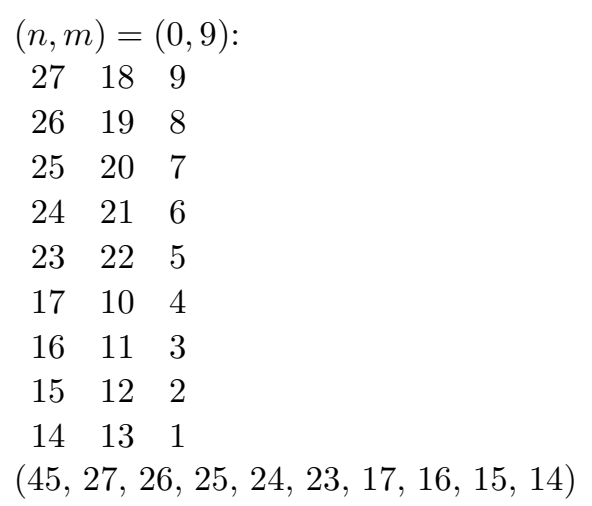

$(n, m)=(1,2)$ :

$\begin{array}{ll}6 & 2\end{array}$

$\begin{array}{lll}7 & 1 & 5\end{array}$

$\begin{array}{lll}8 & 3 & 4\end{array}$

$(11,8,7,6)$
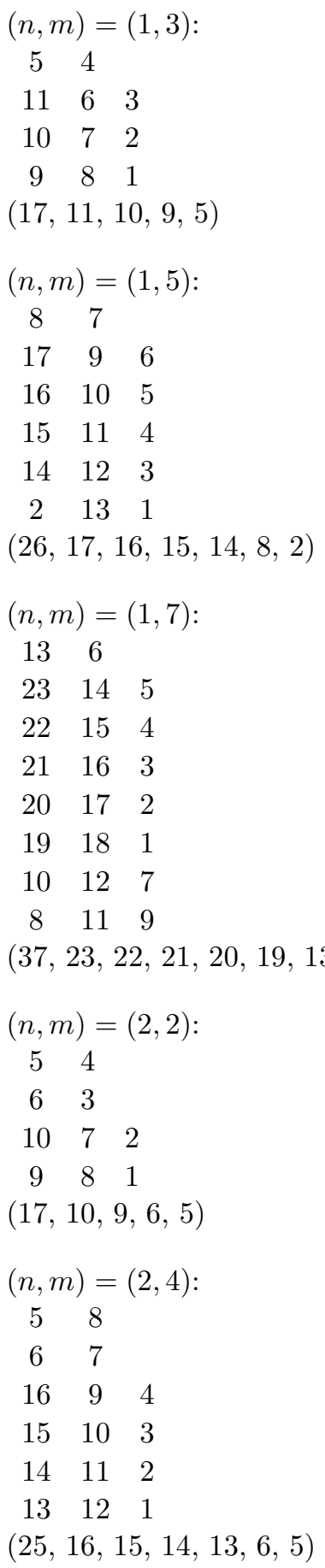


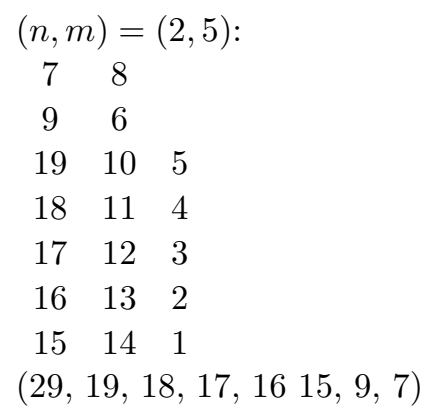

$(29,17,16,15,11,10,9,7)$

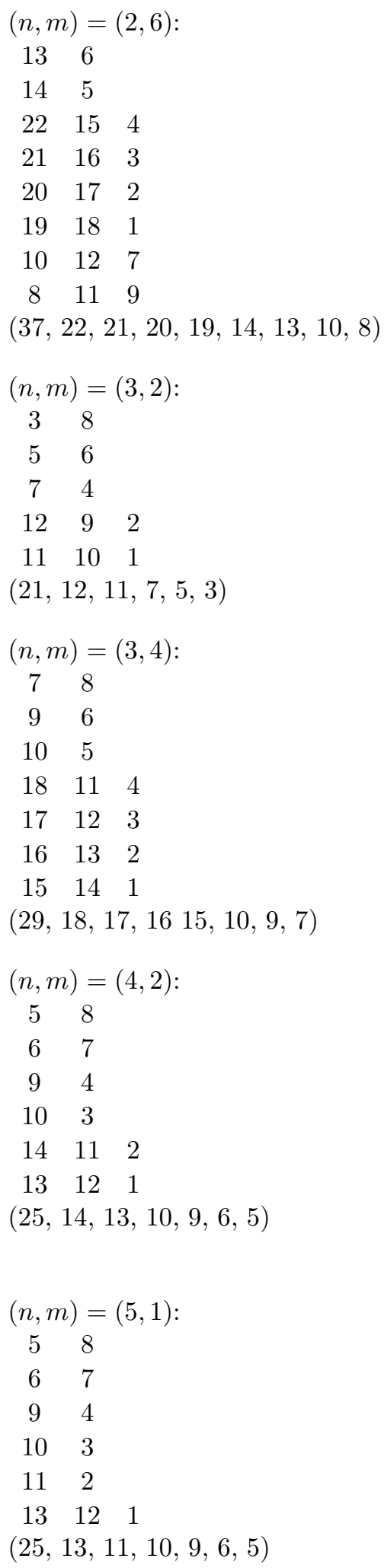




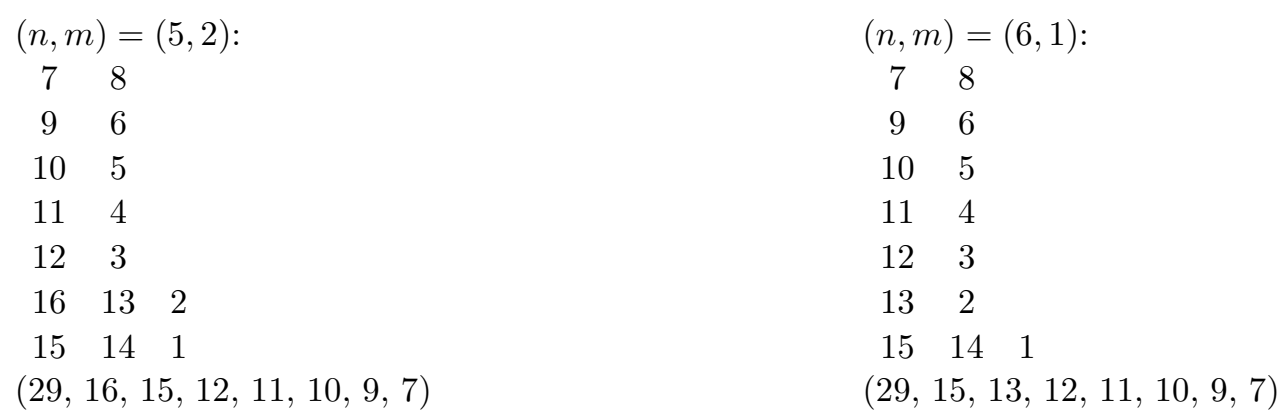

\section{References}

[1] S. Arumugam, Y.C. Lee, K. Premalatha, T.M. Wang, On local antimagic vertex coloring for corona products of graphs, (2018) arXiv:1808.04956v1.

[2] Arumugam S., Premalatha K., Bacă M., Semaničová-Feňovčíková, A.: Local antimagic vertex coloring of a graph. Graphs and Combin. 33, 275-285 (2017).

[3] Lau G.C., Ng H.K., Shiu W.C.: Affirmative solutions on local antimagic chromatic number. Graphs and Combinatorics, (online 2020). https://doi.org/10.1007/s00373-020-02197-2

[4] Lau G.C., Shiu W.C., Ng H.K., : On local antimagic chromatic number of cycle-related join graphs. Discuss. Math. Graph Theory, (online 2018). https://doi:10.7151/dmgt.2177.

[5] Lau G.C., Shiu W.C., Ng H.K.: On local antimagic chromatic number of graphs with cut-vertices, (2020), arXiv:1805.04801.

[6] Lau G.C., Shiu W.C., Ng H.K.: On number of pendants in local antimagic chromatic number, submitted (2020). 